CRYSTALLOGRAPHIC COMMUNICATIONS

ISSN 2056-9890

Received 10 December 2020

Accepted 12 January 2021

Edited by O. Blacque, University of Zürich, Switzerland

Keywords: crystal structure; NSAIDs; sodium 2(1-methyl-5-(4-methylbenzoyl)-1H-pyrrol-2yl)acetate dihydrate; tolmetin sodium.

CCDC reference: 2055407

Supporting information: this article has supporting information at journals.iucr.org/e

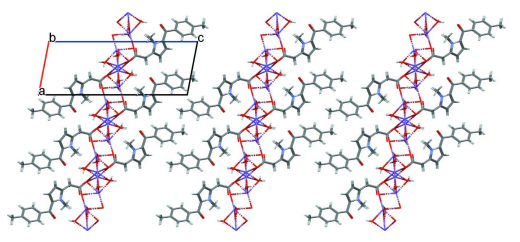

\section{Chemical context}

Non-steroidal anti-inflammatory drugs (NSAIDs) are the gold standard for the management of acute or moderate pain associated with inflammatory changes or trauma (Klippel et al., 2010). These drugs can suppress inflammation, lower body temperature, and reduce pain. In terms of the scale and frequency of use of NSAIDs, they rank first in the world. The combination of analgesic, anti-inflammatory and antipyretic effects determines the advantage of NSAIDs over other pain relievers. Tolmetin, which is one of the most widely used NSAIDs, belongs to the class of hetarylacetic acids (Moreland, 2004; McEvoy, 2007). It is commonly used for the treatment of rheumatoid arthritis, osteoarthritis, ankylosing spondylitis and periarticular disorders. Tolmetin sodium (CAS Number 6449092-2) is the sodium salt form of tolmetin with analgesic, antiinflammatory and antipyretic activities (Cordrey, 1976). In addition, the anticancer activity of Tolmetin has been studied and it was reported that tolmetin has effects on increasing the cytotoxic activity of anti-cancer drugs (Duffy et al., 1998). It inhibits the function of $\beta$-catenin, so tolmetin can be used to develop new anti-cancer agents (Lu et al., 2005).<smiles>Cc1ccc(C(=O)c2ccc(CC(=O)[OH2+])n2C)cc1</smiles>
dimensional fingerprint plots were used to analyze the intermolec present in the crystal.

\title{
Crystal structure of the non-steroidal anti-inflam- matory drug (NSAID) tolmetin sodium
}

\author{
Irina S. Konovalova, ${ }^{a *}$ Sergiy M. Kovalenko, ${ }^{b}$ Dmitry V. Kravchenko ${ }^{c}$ and
}

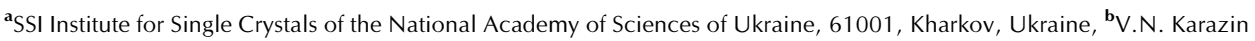
The asymmetric unit of the title compound, sodium 2-[1-methyl-5-(4-methylbenzoyl)-1H-pyrrol-2-yl]acetate dihydrate, $\mathrm{Na}^{+} \cdot \mathrm{C}_{15} \mathrm{H}_{14} \mathrm{NO}_{3}{ }^{-} \cdot 2 \mathrm{H}_{2} \mathrm{O}$, contains two sodium cations, two organic anions $(A$ and $B$ ) and two water molecules. two bridging anions coordinated by the $\mathrm{O}$ atoms of the deprotonated carboxylic groups, and each sodium atom is coordinated by an $\mathrm{O}$ atom of a third anion, which connects pairs of sodium atoms, and a water molecule. As a result, a two- 


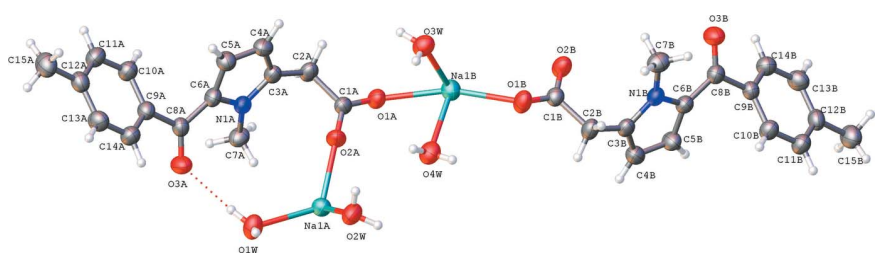

Figure 1

The molecular structure of the title compound showing the atom-labeling scheme. Displacement ellipsoids are drawn at the $50 \%$ probability level.

Recently, work has appeared on the use of this well-known active pharmaceutical compound tolmetin sodium for the development of new dosage forms, for example, novel rectal mucoadhesive hydrogels (Ramadan et al., 2018), thermosensitive mucoadhesive liquid suppositories for rectal delivery (Akl et al., 2019) and different topical gel formulations (Auda et al., 2015).

However, to date, the crystal structure of the substance tolmetin sodium has not been studied and described. Knowledge of the spatial structure of the crystal form of the active pharmaceutical compound is very important to ensure the quality and bioavailability of the drug and, according to the latest pharmacopoeia requirements, X-ray diffraction studies are mandatory for pharmaceutical development. In this work, we carried out an X-ray structural analysis of the crystal form of the substance tolmetin sodium and filled the gap in these studies.

\section{Structural commentary}

The sodium salt of the $\mathrm{C}_{15} \mathrm{H}_{14} \mathrm{NO}_{3}$ organic anion exists in the crystal as a 1:2 hydrate (Fig. 1). The asymmetric unit contains two sodium cations, two organic anions $(A$ and $B)$ and two water molecules. The coordination geometry around the
Table 1

Hydrogen-bond geometry $\left(\AA,^{\circ}\right)$.

\begin{tabular}{lllll}
\hline$D-\mathrm{H} \cdots A$ & $D-\mathrm{H}$ & $\mathrm{H} \cdots A$ & $D \cdots A$ & $D-\mathrm{H} \cdots A$ \\
\hline $\mathrm{O} 1 W-\mathrm{H} 1 W A \cdots \mathrm{O} 3 A$ & 0.89 & 2.12 & $3.002(3)$ & 169 \\
$\mathrm{O} 1 W-\mathrm{H} 1 W B \cdots \mathrm{O} 1 A^{\mathrm{i}}$ & 0.89 & 2.00 & $2.665(4)$ & 130 \\
$\mathrm{O} 2 W-\mathrm{H} 2 W A \cdots \mathrm{O} 2 B^{\mathrm{i}}$ & 0.89 & 1.88 & $2.741(3)$ & 161 \\
$\mathrm{O} 2 W-\mathrm{H} 2 W B \cdots \mathrm{O} 2 B^{\mathrm{ii}}$ & 0.89 & 2.13 & $3.019(3)$ & 172 \\
$\mathrm{O} 3 W-\mathrm{H} 3 W A \cdots \mathrm{O} 3 B^{i i i}$ & 0.89 & 2.16 & $2.961(3)$ & 150 \\
$\mathrm{O} 3 W-\mathrm{H} 3 W B \cdots \mathrm{O} 1 B^{\mathrm{ii}}$ & 0.89 & 1.84 & $2.699(4)$ & 161 \\
$\mathrm{O} 4 W-\mathrm{H} 4 W A \cdots \mathrm{O} 1 B^{\mathrm{ii}}$ & 0.89 & 2.19 & $2.894(3)$ & 136 \\
$\mathrm{O} 4 W-\mathrm{H} 4 W B \cdots \mathrm{O} 3 W^{\mathrm{ii}}$ & 0.89 & 2.02 & $2.889(3)$ & 163 \\
\hline
\end{tabular}

Symmetry codes: (i) $x+1, y, z$; (ii) $-x+1,-y,-z+1$; (iii) $-x,-y,-z+1$.

sodium cations corresponds to a distorted octahedron. Each pair of sodium cations $(A-A$ or $B-B)$ is chelated by two bridging anions coordinated by the $\mathrm{O}$ atoms of the deprotonated carboxylic groups, and each sodium atom is coordinated by an $\mathrm{O}$ atom of a third anion, which connects pairs of sodium atoms, and a water molecule. As a result, a two-dimensional polymer is formed in the crystal (Fig. 2). The $\mathrm{Na}-\mathrm{O}_{\text {anion }}$ distances are 2.298 (2), 2.416 (2) and 2.441 (2) $\AA$ while the $\mathrm{Na}-\mathrm{O}_{\text {water }}$ distances are on average slightly longer, being in the range 2.364 (2)-2.607 (3) $\AA$. It is worth noting that the terminal atom $\mathrm{O} 2 B$ does not interact with a sodium cation.

The analysis of the molecular structure of the anions showed that the terminal $\mathrm{C} 1-\mathrm{O} 1$ and $\mathrm{C} 1-\mathrm{O} 2$ bonds [1.249 (3) and $1.250(3) \AA$ in anion $A, 1.243$ (3) and 1.250 (3) $\AA$ in anion $B$ ] are very similar to each other and are slightly elongated in comparison with the standard value of $1.210 \AA$ of a carbonyl group (Burgi et al., 1994). It is also much shorter than the standard $\mathrm{C}-\mathrm{O}$ single bond observed for a hydroxyl group $(1.362 \AA)$. We can assume that the negative charge is delocalized on both terminal $\mathrm{O}$ atoms for each anion.

The toluene substituent is in a synperiplanar conformation with respect to the $\mathrm{C} 5-\mathrm{C} 6$ bond of the pyrrole ring: the $\mathrm{C} 5-$

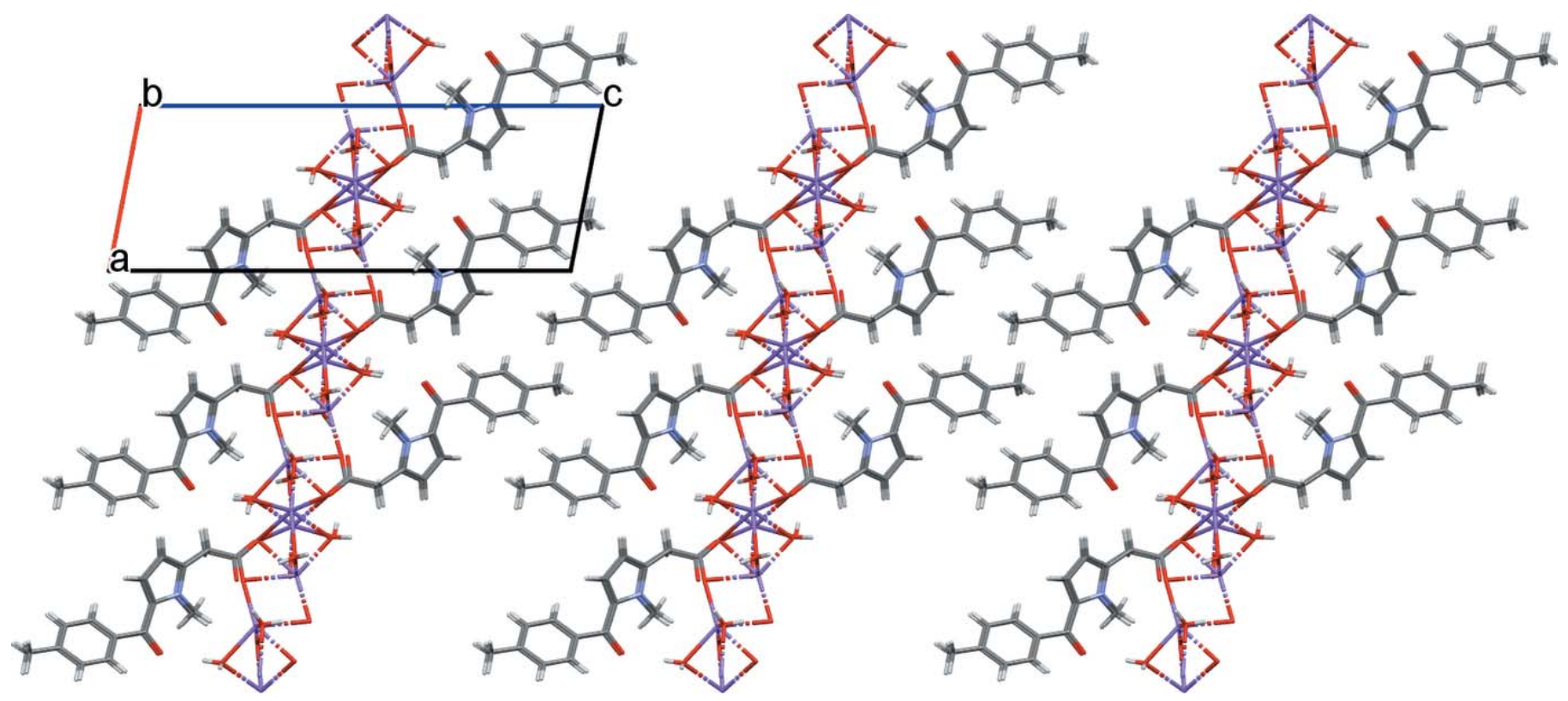

Figure 2

Two-dimensional polymeric chains in the crystal of the title compound. 


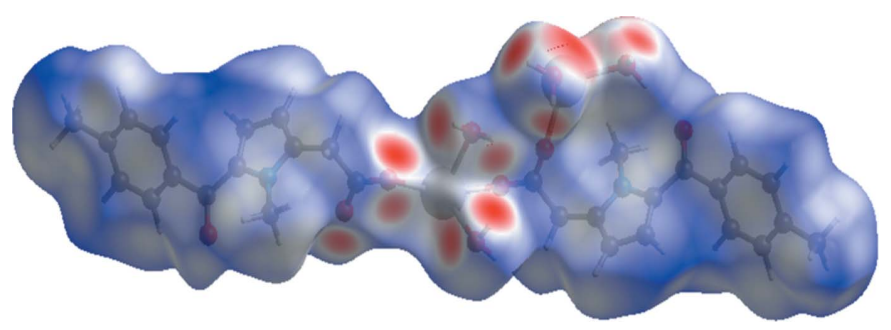

Figure 3

The Hirshfeld surface of the title compound mapped over $d_{\text {norm }}$.

C6-C $8-\mathrm{C} 9$ torsion angle is $26.9(4)^{\circ}$ in molecule $A$ and $-29.0(4)^{\circ}$ in molecule $B$. The relative orientation of the toluene ring with respect to the pyrrole ring, neither planar nor perpendicular, is given by the the $\mathrm{C} 6-\mathrm{C} 8-\mathrm{C} 9-\mathrm{C} 10$ torsion angle: $41.3(4)^{\circ}$ in molecule $A$ and $-38.2(4)^{\circ}$ in molecule $B$. Such an orientation mainly minimizes the intermolecular $\mathrm{H} \cdot \mathrm{H}$ H repulsions.

\section{Supramolecular features}

In the crystal, $\mathrm{O}-\mathrm{H} \cdots \mathrm{O}$ hydrogen bonds (Table 1) are formed between $\mathrm{H}$ atoms of the water molecules (donors) and $\mathrm{O}$ atoms of the anions (acceptors), forming a two-dimensional network parallel to (001).

\section{Hirshfeld surface analysis}

Crystal Explorer 17.5 (Turner et al., 2017) was used to analyze the interactions in the crystal: fingerprint plots mapped over $d_{\text {norm }}$ (Figs. 3 and 4) were generated. The molecular Hirshfeld surfaces were obtained using a standard (high) surface resolution with the three-dimensional $d_{\text {norm }}$ surfaces mapped over a fixed color scale of -0.666 (red) to 1.384 (blue). The areas colored red on the $d_{\text {norm-mapped Hirshfeld surfaces (Fig. 3) }}$ correspond to the contacts which are shorter than van der Waals radii sum of the closest atoms. As can be seen in Fig. 4, short contacts are present at the hydrogen atoms and oxygen lone pair of the water molecules. In addition, the areas of short
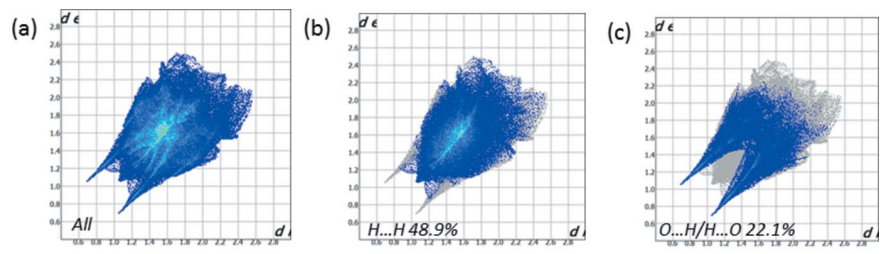

(d)

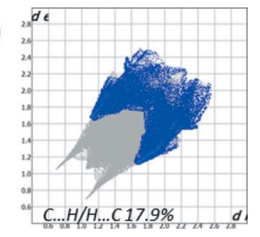

(e)

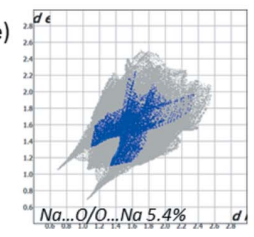

Figure 4

(a) The two-dimensional fingerprint plot for the title compound, and those delineated into $(b) \mathrm{H} \cdots \mathrm{H}(48.9 \%),(c) \mathrm{O} \cdots \mathrm{H} / \mathrm{H} \cdots \mathrm{O}(22.1 \%),(d)$ $\mathrm{C} \cdot \mathrm{H} / \mathrm{H} \cdots \mathrm{C}(17.9 \%)$ and $(e) \mathrm{Na} \cdot \mathrm{O} / \mathrm{O} \cdots \mathrm{Na}(5.4 \%)$ contacts. contacts are located at the oxygen atoms of carbonyl groups (Fig. 3).

All the intermolecular interactions of the title compound are shown in the two-dimensional fingerprint plot presented in Fig. 4. The contribution of the $\mathrm{O} \cdots \mathrm{H} / \mathrm{H} \cdots \mathrm{O}$ contacts, corresponding to the $\mathrm{O}-\mathrm{H} \cdots \mathrm{O}$ interaction, is represented by a pair of long sharp spikes $(22.1 \%)$. This indicates that $\mathrm{O}-$ $\mathrm{H}$... O hydrogen bonds are the strongest interactions in the crystal of the title compound (Fig. 4).

\section{Database survey}

A search of the Cambridge Structural Database (CSD, Version 5.41, update of November 2019; Groom et al., 2016) for the 2-(1-methyl-5-(4-methylbenzoyl)-1H-pyrrol-2-yl)acetate skeleton yielded only two hits, 2-methoxyphenyl 2-\{2-[1methyl-5-(4-methylbenzoyl)pyrrol-2-yl]acetamido\}acetate (CSD refcode MODNID; Lou et al., 2008) and bis(dimethylsulfoxide- $O)$ tetrakis $\left[\mu_{2}-1\right.$-methyl-5-( $p$-toluoyl $)-1 H$-pyrrole-2acetato- $\left.O, O^{\prime}\right] \operatorname{dicopper}(\mathrm{II})$ (SETBIC; Dendrinou-Samara et al., 1990).

\section{Crystallization}

Crystallization by slow evaporation of an aqueous solution of tolmetin sodium was carried out to provide colorless blockshaped single crystals suitable for a X-ray diffraction analysis (Fig. 5).

\section{Refinement}

Crystal data, data collection and structure refinement details are summarized in Table $2 . \mathrm{H}$ atoms were placed in calculated positions $(\mathrm{O}-\mathrm{H}=0.98 \AA, \mathrm{C}-\mathrm{H}=0.93-0.96 \AA)$ and refined as riding with $U_{\text {iso }} \mathrm{H}=1.2 U_{\text {eq }}(\mathrm{C})$ or $1.5 U_{\text {eq }}(\mathrm{O}, \mathrm{C}$-methyl $)$.

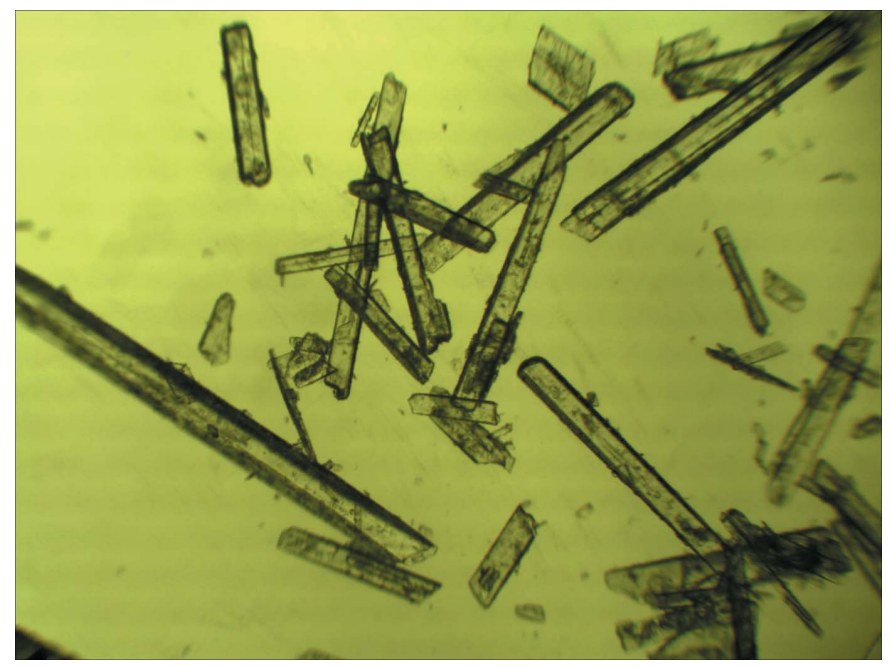

Figure 5

Crystals of tolmetin sodium. 
Table 2

Experimental details.

\begin{tabular}{|c|c|}
\hline \multicolumn{2}{|l|}{ Crystal data } \\
\hline Chemical formula & $2 \mathrm{Na}^{+} \cdot 2 \mathrm{C}_{15} \mathrm{H}_{14} \mathrm{NO}_{3}^{-} \cdot 4 \mathrm{H}_{2} \mathrm{O}$ \\
\hline$M_{\mathrm{r}}$ & 630.59 \\
\hline Crystal system, space group & Triclinic, $P \overline{1}$ \\
\hline Temperature (K) & 293 \\
\hline$a, b, c(\AA)$ & 8.5404 (8), $9.0144(9), 21.6217$ (19) \\
\hline$\alpha, \beta, \gamma\left({ }^{\circ}\right)$ & $92.922(8), 98.873(7), 113.038(9)$ \\
\hline$V\left(\AA^{3}\right)$ & $1502.1(3)$ \\
\hline$Z$ & 2 \\
\hline Radiation type & Мо $K \alpha$ \\
\hline$\mu\left(\mathrm{mm}^{-1}\right)$ & 0.13 \\
\hline Crystal size $(\mathrm{mm})$ & $0.4 \times 0.2 \times 0.1$ \\
\hline \multicolumn{2}{|l|}{ Data collection } \\
\hline Diffractometer & $\begin{array}{l}\text { Rigaku Oxford Diffraction } \\
\text { Xcalibur, Sapphire3 }\end{array}$ \\
\hline Absorption correction & $\begin{array}{l}\text { Multi-scan (CrysAlis PRO; Rigaku } \\
\text { OD, 2018) }\end{array}$ \\
\hline$T_{\min }, T_{\max }$ & $0.737,1.000$ \\
\hline $\begin{array}{l}\text { No. of measured, independent and } \\
\text { observed }[I>2 \sigma(I)] \text { reflections }\end{array}$ & $11985,5283,3288$ \\
\hline$R_{\text {int }}$ & 0.060 \\
\hline$(\sin \theta / \lambda)_{\max }\left(\AA^{-1}\right)$ & 0.595 \\
\hline \multicolumn{2}{|l|}{ Refinement } \\
\hline$R\left[F^{2}>2 \sigma\left(F^{2}\right)\right], w R\left(F^{2}\right), S$ & $0.064,0.191,1.00$ \\
\hline No. of reflections & 5283 \\
\hline No. of parameters & 403 \\
\hline $\mathrm{H}$-atom treatment & $\mathrm{H}$-atom parameters constrained \\
\hline$\Delta \rho_{\max }, \Delta \rho_{\min }\left(\mathrm{e} \AA^{-3}\right)$ & $0.38,-0.28$ \\
\hline
\end{tabular}

Computer programs: CrysAlis PRO (Rigaku OD, 2018), SHELXT (Sheldrick, 2015a), SHELXL (Sheldrick, 2015b) and OLEX2 (Dolomanov et al., 2009).

\section{References}

Akl, M. A., Ismael, H. R., Abd Allah, F. I., Kassem, A. A. \& Samy, A. M. (2019). Drug Dev. Ind. Pharm. 45, 252-264.
Auda, S. H., El-Rasoul, S. A., Ahmed, M. M., Osman, S. K. \& ElBadry, M. (2015). J. Pharm. Investig. 45, 311-317.

Burgi, H.-B. \& Dunitz, J. D. (1994). Structure Correlation, vol. 2, pp. 741-784. Weinheim: VCH.

Cordrey, L. J. (1976). J. Am. Geriatr. Soc. 24, 440-446.

Dendrinou-Samara, C., Kessissoglou, D. P., Manoussakis, G. E., Mentzafos, D. \& Terzis, A. (1990). J. Chem. Soc. Dalton Trans. pp. 959-965.

Dolomanov, O. V., Bourhis, L. J., Gildea, R. J., Howard, J. A. K. \& Puschmann, H. (2009). J. Appl. Cryst. 42, 339-341.

Duffy, C. P., Elliott, C. J., O'Connor, R. A., Heenan, M. M., Coyle, S., Cleary, I. M., Kavanagh, K., Verhaegen, S., O'Loughlin, C. M., NicAmhlaoibh, R. \& Clynes, M. (1998). Eur. J. Cancer, 34, 12501259.

Groom, C. R., Bruno, I. J., Lightfoot, M. P. \& Ward, S. C. (2016). Acta Cryst. B72, 171-179.

Klippel, J. H., Stone, J. H., Crofford, L. J. \& White, P. H. (2010). Nonsteroidal Anti-Inflammatory Drugs. In The Pocket Primer on the Rheumatic Diseases. London: Springer.

Lou, B.-Y., Guo, X. \& Lin, Q. (2008). Acta Cryst. E64, o1439.

Lu, D., Cottam, H. B., Corr, M. \& Carson, D. A. (2005). Proc. Natl Acad. Sci. USA, 102, 18567-18571.

McEvoy, G. K. (2007). Editor. AHFS Drug Information, pp. 21182122. Bethesda, MD: American Society of Health-System Pharmacists.

Moreland, L. W. (2004). Tolmetin. Rheumatology and Immunology Therapy. Berlin, Heidelberg: Springer.

Ramadan, A. A., Elbakry, A. M., Esmaeil, A. H. \& Khaleel, S. A. (2018). J. Pharm. Investig. 48, 673-683.

Rigaku OD (2018). CrysAlis PRO. Rigaku Oxford Diffraction Ltd, Yarnton, England.

Sheldrick, G. M. (2015a). Acta Cryst. A71, 3-8.

Sheldrick, G. M. (2015b). Acta Cryst. C71, 3-8.

Turner, M. J., McKinnon, J. J., Wolff, S. K., Grimwood, D. J., Spackman, P. R., Jayatilaka, D. \& Spackman, M. A. (2017). Crystal Explorer 17. University of Western Australia. http:// hirshfeldsurface.net. 


\title{
supporting information
}

Acta Cryst. (2021). E77, 134-137 [https://doi.org/10.1107/S2056989021000414]

\section{Crystal structure of the non-steroidal anti-inflammatory drug (NSAID) tolmetin sodium}

\author{
Irina S. Konovalova, Sergiy M. Kovalenko, Dmitry V. Kravchenko and Vladimir P. Chuev \\ Computing details
}

Data collection: CrysAlis PRO (Rigaku OD, 2018); cell refinement: CrysAlis PRO (Rigaku OD, 2018); data reduction: CrysAlis PRO (Rigaku OD, 2018); program(s) used to solve structure: ShelXT (Sheldrick, 2015a); program(s) used to refine structure: SHELXL (Sheldrick, 2015b); molecular graphics: OLEX2 (Dolomanov et al., 2009); software used to prepare material for publication: OLEX2 (Dolomanov et al., 2009).

Sodium 2-[1-methyl-5-(4-methylbenzoyl)-1H-pyrrol-2-yl]acetate dihydrate

\section{Crystal data}

$2 \mathrm{Na}^{+} \cdot 2 \mathrm{C}_{15} \mathrm{H}_{14} \mathrm{NO}_{3}{ }^{-} \cdot 4 \mathrm{H}_{2} \mathrm{O}$

$M_{r}=630.59$

Triclinic, $P \overline{1}$

$a=8.5404(8) \AA$

$b=9.0144(9) \AA$

$c=21.6217(19) \AA$

$\alpha=92.922(8)^{\circ}$

$\beta=98.873(7)^{\circ}$

$\gamma=113.038(9)^{\circ}$

$V=1502.1(3) \AA^{3}$

\section{Data collection}

Rigaku Oxford Diffraction Xcalibur, Sapphire3 diffractometer

Radiation source: fine-focus sealed X-ray tube, Enhance (Mo) X-ray Source

Graphite monochromator

Detector resolution: 16.1827 pixels $\mathrm{mm}^{-1}$

phi and $\omega$ scans

Absorption correction: multi-scan

(CrysAlisPro; Rigaku OD, 2018)

\section{Refinement}

Refinement on $F^{2}$

Least-squares matrix: full

$R\left[F^{2}>2 \sigma\left(F^{2}\right)\right]=0.064$

$w R\left(F^{2}\right)=0.191$

$S=1.00$

5283 reflections

403 parameters
$Z=2$

$F(000)=664$

$D_{\mathrm{x}}=1.394 \mathrm{Mg} \mathrm{m}^{-3}$

Mo $K \alpha$ radiation, $\lambda=0.71073 \AA$

Cell parameters from 1420 reflections

$\theta=3.1-24.2^{\circ}$

$\mu=0.13 \mathrm{~mm}^{-1}$

$T=293 \mathrm{~K}$

Block, colourless

$0.4 \times 0.2 \times 0.1 \mathrm{~mm}$

$T_{\min }=0.737, T_{\max }=1.000$

11985 measured reflections

5283 independent reflections

3288 reflections with $I>2 \sigma(I)$

$R_{\text {int }}=0.060$

$\theta_{\max }=25.0^{\circ}, \theta_{\min }=2.9^{\circ}$

$h=-10 \rightarrow 10$

$k=-10 \rightarrow 10$

$l=-25 \rightarrow 25$

0 restraints

Primary atom site location: inferred from neighbouring sites

Hydrogen site location: difference Fourier map

$\mathrm{H}$-atom parameters constrained

$w=1 /\left[\sigma^{2}\left(F_{\mathrm{o}}{ }^{2}\right)+(0.0844 P)^{2}\right]$

where $P=\left(F_{\mathrm{o}}^{2}+2 F_{\mathrm{c}}^{2}\right) / 3$ 
$(\Delta / \sigma)_{\max }<0.001$

$\Delta \rho_{\min }=-0.28$ e $\AA^{-3}$

$\Delta \rho_{\max }=0.38 \mathrm{e} \AA^{-3}$

Special details

Geometry. All esds (except the esd in the dihedral angle between two 1.s. planes) are estimated using the full covariance matrix. The cell esds are taken into account individually in the estimation of esds in distances, angles and torsion angles; correlations between esds in cell parameters are only used when they are defined by crystal symmetry. An approximate (isotropic) treatment of cell esds is used for estimating esds involving l.s. planes.

Fractional atomic coordinates and isotropic or equivalent isotropic displacement parameters $\left(\AA^{2}\right)$

\begin{tabular}{|c|c|c|c|c|}
\hline & $x$ & $y$ & $z$ & $U_{\text {iso }} * / U_{\text {eq }}$ \\
\hline $\mathrm{Na} 1 \mathrm{~A}$ & $1.15418(14)$ & $0.48055(14)$ & $0.46665(5)$ & $0.0463(4)$ \\
\hline O1A & $0.6328(3)$ & $0.4448(3)$ & $0.43538(9)$ & $0.0463(5)$ \\
\hline $\mathrm{O} 2 \mathrm{~A}$ & $0.8756(3)$ & $0.4310(3)$ & $0.41918(9)$ & $0.0527(6)$ \\
\hline $\mathrm{O} 3 \mathrm{~A}$ & $1.3144(3)$ & $0.5970(3)$ & $0.27170(10)$ & $0.0572(6)$ \\
\hline $\mathrm{N} 1 \mathrm{~A}$ & $0.9595(3)$ & $0.5322(3)$ & $0.28684(10)$ & $0.0370(6)$ \\
\hline $\mathrm{C} 1 \mathrm{~A}$ & $0.7367(4)$ & $0.4452(3)$ & $0.40052(13)$ & $0.0369(7)$ \\
\hline $\mathrm{C} 2 \mathrm{~A}$ & $0.6907(4)$ & $0.4728(4)$ & $0.33229(13)$ & $0.0441(7)$ \\
\hline $\mathrm{H} 2 \mathrm{AA}$ & 0.568753 & 0.405386 & 0.316973 & $0.053^{*}$ \\
\hline $\mathrm{H} 2 \mathrm{AB}$ & 0.705297 & 0.584995 & 0.332112 & $0.053^{*}$ \\
\hline $\mathrm{C} 3 \mathrm{~A}$ & $0.7899(4)$ & $0.4394(4)$ & $0.28655(13)$ & $0.0397(7)$ \\
\hline $\mathrm{C} 4 \mathrm{~A}$ & $0.7319(4)$ & $0.3165(4)$ & $0.23764(14)$ & $0.0445(7)$ \\
\hline $\mathrm{H} 4 \mathrm{~A}$ & 0.620599 & 0.234969 & 0.227154 & $0.053^{*}$ \\
\hline $\mathrm{C} 5 \mathrm{~A}$ & $0.8671(4)$ & $0.3346(4)$ & $0.20653(14)$ & $0.0446(7)$ \\
\hline $\mathrm{H} 5 \mathrm{~A}$ & 0.862808 & 0.267733 & 0.171513 & $0.053^{*}$ \\
\hline C6A & $1.0108(4)$ & $0.4710(4)$ & $0.23717(13)$ & $0.0403(7)$ \\
\hline C7A & $1.0633(4)$ & $0.6845(3)$ & $0.32769(13)$ & $0.0453(7)$ \\
\hline H7AA & 1.124646 & 0.663236 & 0.364871 & $0.068^{*}$ \\
\hline $\mathrm{H} 7 \mathrm{AB}$ & 0.988702 & 0.733150 & 0.339497 & $0.068^{*}$ \\
\hline $\mathrm{H} 7 \mathrm{AC}$ & 1.144888 & 0.757182 & 0.305536 & $0.068^{*}$ \\
\hline $\mathrm{C} 8 \mathrm{~A}$ & $1.1889(4)$ & $0.5209(4)$ & $0.22929(13)$ & $0.0392(7)$ \\
\hline C9A & $1.2180(4)$ & $0.4679(3)$ & $0.16703(13)$ & $0.0399(7)$ \\
\hline $\mathrm{C} 10 \mathrm{~A}$ & $1.1169(4)$ & $0.4703(4)$ & $0.11027(14)$ & $0.0457(7)$ \\
\hline H10A & 1.026896 & 0.503734 & 0.110630 & $0.055^{*}$ \\
\hline C11A & $1.1493(4)$ & $0.4236(4)$ & $0.05367(14)$ & $0.0488(8)$ \\
\hline H11A & 1.079818 & 0.425219 & 0.016307 & $0.059^{*}$ \\
\hline $\mathrm{C} 12 \mathrm{~A}$ & $1.2836(4)$ & $0.3741(4)$ & $0.05106(15)$ & $0.0455(8)$ \\
\hline $\mathrm{C} 13 \mathrm{~A}$ & $1.3832(4)$ & $0.3726(4)$ & $0.10725(15)$ & $0.0508(8)$ \\
\hline H13A & 1.472156 & 0.337798 & 0.106762 & $0.061^{*}$ \\
\hline $\mathrm{C} 14 \mathrm{~A}$ & $1.3541(4)$ & $0.4217(4)$ & $0.16450(15)$ & $0.0478(8)$ \\
\hline $\mathrm{H} 14 \mathrm{~A}$ & 1.426516 & 0.423803 & 0.201665 & $0.057^{*}$ \\
\hline $\mathrm{C} 15 \mathrm{~A}$ & $1.3099(5)$ & $0.3182(4)$ & $-0.01169(16)$ & $0.0620(9)$ \\
\hline H15A & 1.253192 & 0.201892 & -0.019774 & $0.093 *$ \\
\hline H15B & 1.431599 & 0.351811 & -0.011128 & $0.093^{*}$ \\
\hline $\mathrm{H} 15 \mathrm{C}$ & 1.261676 & 0.365083 & -0.044267 & $0.093^{*}$ \\
\hline Na1B & $0.45480(15)$ & $0.23476(14)$ & $0.49496(5)$ & $0.0497(4)$ \\
\hline O1B & $0.3539(3)$ & $0.0551(3)$ & $0.57285(10)$ & $0.0520(6)$ \\
\hline $\mathrm{O} 2 \mathrm{~B}$ & 0.1279 & $0.0952(3)$ & $0.59464(9)$ & $0.0517(6)$ \\
\hline
\end{tabular}




\begin{tabular}{|c|c|c|c|c|}
\hline $\mathrm{O} 3 \mathrm{~B}$ & $-0.3202(3)$ & $-0.0817(3)$ & $0.73382(10)$ & $0.0561(6)$ \\
\hline N1B & $0.0316(3)$ & -0.0303 & $0.72107(10)$ & $0.0392(6)$ \\
\hline $\mathrm{C} 1 \mathrm{~B}$ & $0.2576(4)$ & 0.0630 & $0.60931(13)$ & $0.0372(7)$ \\
\hline $\mathrm{C} 2 \mathrm{~B}$ & $0.2993(4)$ & $0.0232(4)$ & $0.67578(13)$ & $0.0469(8)$ \\
\hline $\mathrm{H} 2 \mathrm{BA}$ & 0.277121 & -0.091173 & 0.673239 & $0.056^{*}$ \\
\hline $\mathrm{H} 2 \mathrm{BB}$ & 0.422204 & 0.084105 & 0.691795 & $0.056^{*}$ \\
\hline C3B & 0.2033 (4) & $0.0571(4)$ & $0.72214(13)$ & $0.0405(7)$ \\
\hline $\mathrm{C} 4 \mathrm{~B}$ & $0.2635(4)$ & 0.1815 & 0.77089 (14) & $0.0505(8)$ \\
\hline H4B & 0.375714 & 0.261529 & 0.781418 & $0.061^{*}$ \\
\hline C5B & $0.1287(4)$ & 0.1671 (4) & $0.80151(14)$ & $0.0451(7)$ \\
\hline H5B & 0.134436 & 0.234532 & 0.836595 & $0.054 *$ \\
\hline $\mathrm{C} 6 \mathrm{~B}$ & $-0.0169(4)$ & $0.0342(4)$ & $0.77058(13)$ & $0.0414(7)$ \\
\hline C7B & $-0.0783(4)$ & -0.1782 & $0.67844(14)$ & $0.0505(8)$ \\
\hline H7BA & -0.140894 & -0.151452 & 0.643088 & $0.076^{*}$ \\
\hline H7BB & -0.158712 & -0.252622 & 0.700442 & $0.076^{*}$ \\
\hline $\mathrm{H} 7 \mathrm{BC}$ & -0.007306 & -0.227504 & 0.663767 & $0.076^{*}$ \\
\hline $\mathrm{C} 8 \mathrm{~B}$ & $-0.1949(4)$ & -0.0101 & $0.77748(14)$ & $0.0423(7)$ \\
\hline C9B & $-0.2254(4)$ & 0.0398 & $0.83992(13)$ & $0.0396(7)$ \\
\hline $\mathrm{C} 10 \mathrm{~B}$ & $-0.1236(4)$ & $0.0352(4)$ & 0.89619 (14) & $0.0458(8)$ \\
\hline H10B & -0.034555 & 0.000647 & 0.895357 & $0.055^{*}$ \\
\hline C11B & $-0.1552(4)$ & 0.0819 & $0.95293(14)$ & $0.0483(8)$ \\
\hline H11B & -0.086237 & 0.078848 & 0.990196 & $0.058^{*}$ \\
\hline C12B & -0.2879 & $0.1337(4)$ & $0.95586(14)$ & $0.0463(8)$ \\
\hline C13B & $-0.3882(4)$ & $0.1359(4)$ & $0.89975(15)$ & $0.0505(8)$ \\
\hline H13B & -0.477015 & 0.170816 & 0.900353 & $0.061^{*}$ \\
\hline C14B & $-0.3588(4)$ & $0.0871(4)$ & $0.84272(15)$ & $0.0473(8)$ \\
\hline H14B & -0.430573 & 0.086230 & 0.805569 & $0.057^{*}$ \\
\hline C15B & $-0.3154(4)$ & $0.1885(5)$ & $1.01896(16)$ & $0.0610(9)$ \\
\hline H15D & -0.263611 & 0.144401 & 1.051608 & $0.091^{*}$ \\
\hline H15E & -0.437342 & 0.150939 & 1.018731 & $0.091^{*}$ \\
\hline $\mathrm{H} 15 \mathrm{~F}$ & -0.262690 & 0.304934 & 1.026643 & $0.091 *$ \\
\hline $\mathrm{O} 1 \mathrm{~W}$ & $1.3788(3)$ & $0.5461(3)$ & $0.40784(10)$ & $0.0541(6)$ \\
\hline H1WA & 1.363692 & 0.575550 & 0.369478 & $0.081^{*}$ \\
\hline H1WB & 1.405922 & 0.460320 & 0.406558 & $0.081^{*}$ \\
\hline $\mathrm{O} 2 \mathrm{~W}$ & $1.1457(3)$ & 0.2133 & $0.48044(9)$ & $0.0529(6)$ \\
\hline H2WA & 1.128262 & 0.190038 & 0.518967 & $0.079^{*}$ \\
\hline $\mathrm{H} 2 \mathrm{WB}$ & 1.066822 & 0.127148 & 0.455027 & $0.079^{*}$ \\
\hline $\mathrm{O} 3 \mathrm{~W}$ & $0.3857(3)$ & 0.0443 & $0.40179(10)$ & $0.0522(6)$ \\
\hline H3WA & 0.383694 & 0.094075 & 0.367336 & $0.078^{*}$ \\
\hline H3WB & 0.464794 & 0.002995 & 0.401246 & $0.078^{*}$ \\
\hline $\mathrm{O} 4 \mathrm{~W}$ & $0.7371(3)$ & 0.2175 & $0.52238(10)$ & $0.0538(6)$ \\
\hline H4WA & 0.769350 & 0.168269 & 0.493211 & $0.081^{*}$ \\
\hline H4WB & 0.720981 & 0.138360 & 0.546861 & $0.081^{*}$ \\
\hline
\end{tabular}

Atomic displacement parameters $\left(\AA^{2}\right)$

\begin{tabular}{lllllll}
\hline & $U^{11}$ & $U^{22}$ & $U^{33}$ & $U^{12}$ & $U^{13}$ & $U^{23}$ \\
\hline Na1A & $0.0365(7)$ & $0.0608(8)$ & $0.0418(7)$ & $0.0197(6)$ & $0.0075(5)$ & $0.0091(6)$
\end{tabular}




\begin{tabular}{|c|c|c|c|c|c|c|}
\hline O1A & $0.0426(12)$ & $0.0665(14)$ & $0.0355(12)$ & $0.0258(11)$ & $0.0121(9)$ & $0.0097(10)$ \\
\hline $\mathrm{O} 2 \mathrm{~A}$ & $0.0335(12)$ & $0.0802(16)$ & $0.0450(13)$ & $0.0235(11)$ & $0.0045(9)$ & $0.0188(11)$ \\
\hline $\mathrm{O} 3 \mathrm{~A}$ & $0.0405(13)$ & $0.0796(17)$ & $0.0398(13)$ & $0.0148(12)$ & $0.0031(10)$ & $-0.0030(11)$ \\
\hline N1A & $0.0369(14)$ & $0.0414(13)$ & $0.0326(13)$ & $0.0153(11)$ & $0.0071(10)$ & $0.0057(10)$ \\
\hline $\mathrm{C} 1 \mathrm{~A}$ & $0.0346(16)$ & $0.0406(16)$ & $0.0358(16)$ & $0.0154(13)$ & $0.0062(13)$ & $0.0090(13)$ \\
\hline $\mathrm{C} 2 \mathrm{~A}$ & $0.0430(17)$ & 0.0605 (19) & $0.0377(17)$ & $0.0287(16)$ & $0.0091(13)$ & $0.0145(14)$ \\
\hline $\mathrm{C} 3 \mathrm{~A}$ & $0.0360(16)$ & $0.0535(18)$ & $0.0337(16)$ & $0.0211(14)$ & $0.0083(12)$ & $0.0098(13)$ \\
\hline $\mathrm{C} 4 \mathrm{~A}$ & $0.0381(17)$ & $0.0456(18)$ & 0.0409 (17) & 0.0093 (14) & $0.0030(13)$ & $0.0040(14)$ \\
\hline $\mathrm{C} 5 \mathrm{~A}$ & $0.0436(18)$ & $0.0441(17)$ & $0.0417(17)$ & $0.0138(15)$ & $0.0081(14)$ & $0.0008(14)$ \\
\hline C6A & $0.0422(17)$ & $0.0475(17)$ & $0.0310(16)$ & $0.0164(14)$ & $0.0105(13)$ & $0.0049(13)$ \\
\hline C7A & $0.0509(19)$ & $0.0416(16)$ & $0.0377(17)$ & $0.0149(15)$ & $0.0046(14)$ & $-0.0007(13)$ \\
\hline $\mathrm{C} 8 \mathrm{~A}$ & $0.0367(16)$ & $0.0446(17)$ & $0.0377(17)$ & 0.0158 (14) & $0.0115(13)$ & $0.0092(13)$ \\
\hline C9A & $0.0382(17)$ & $0.0432(17)$ & $0.0344(16)$ & $0.0121(14)$ & $0.0090(12)$ & $0.0016(13)$ \\
\hline $\mathrm{C} 10 \mathrm{~A}$ & $0.0449(18)$ & $0.0544(19)$ & $0.0398(18)$ & $0.0215(16)$ & $0.0087(14)$ & $0.0085(15)$ \\
\hline C11A & $0.052(2)$ & $0.0553(19)$ & $0.0364(17)$ & $0.0195(17)$ & $0.0064(14)$ & $0.0068(14)$ \\
\hline $\mathrm{C} 12 \mathrm{~A}$ & $0.0412(18)$ & $0.0457(18)$ & 0.0473 (19) & $0.0129(15)$ & $0.0147(14)$ & $0.0052(14)$ \\
\hline C13A & $0.0431(18)$ & $0.063(2)$ & $0.050(2)$ & $0.0244(17)$ & $0.0117(15)$ & $0.0034(16)$ \\
\hline $\mathrm{C} 14 \mathrm{~A}$ & $0.0442(18)$ & $0.059(2)$ & $0.0394(17)$ & $0.0204(16)$ & $0.0067(14)$ & $0.0085(15)$ \\
\hline $\mathrm{C} 15 \mathrm{~A}$ & $0.061(2)$ & $0.067(2)$ & $0.055(2)$ & $0.0201(19)$ & $0.0208(17)$ & $-0.0028(17)$ \\
\hline $\mathrm{Na} 1 \mathrm{~B}$ & $0.0459(7)$ & $0.0559(8)$ & $0.0458(7)$ & $0.0174(6)$ & $0.0125(5)$ & $0.0075(6)$ \\
\hline O1B & $0.0549(14)$ & $0.0749(16)$ & $0.0407(12)$ & $0.0366(12)$ & $0.0200(10)$ & $0.0163(11)$ \\
\hline $\mathrm{O} 2 \mathrm{~B}$ & $0.0505(13)$ & $0.0781(16)$ & $0.0399(12)$ & $0.0372(12)$ & $0.0140(10)$ & $0.0148(11)$ \\
\hline $\mathrm{O} 3 \mathrm{~B}$ & $0.0418(13)$ & $0.0753(16)$ & $0.0411(13)$ & $0.0154(12)$ & $0.0042(10)$ & $-0.0022(11)$ \\
\hline N1B & $0.0411(14)$ & $0.0489(14)$ & $0.0304(13)$ & $0.0200(12)$ & $0.0092(10)$ & $0.0062(11)$ \\
\hline $\mathrm{C} 1 \mathrm{~B}$ & $0.0401(17)$ & $0.0379(15)$ & $0.0334(16)$ & $0.0152(13)$ & $0.0087(13)$ & $0.0024(12)$ \\
\hline $\mathrm{C} 2 \mathrm{~B}$ & $0.0497(19)$ & $0.063(2)$ & $0.0358(17)$ & $0.0279(16)$ & $0.0147(14)$ & $0.0139(15)$ \\
\hline $\mathrm{C} 3 \mathrm{~B}$ & $0.0379(17)$ & $0.0560(19)$ & $0.0327(16)$ & $0.0228(15)$ & $0.0081(12)$ & $0.0129(14)$ \\
\hline C4B & $0.0376(17)$ & $0.061(2)$ & 0.0451 (19) & $0.0123(16)$ & $0.0061(14)$ & $0.0113(16)$ \\
\hline $\mathrm{C} 5 \mathrm{~B}$ & $0.0419(17)$ & $0.0538(19)$ & $0.0346(17)$ & $0.0148(15)$ & $0.0076(13)$ & $-0.0031(14)$ \\
\hline C6B & $0.0379(17)$ & $0.0523(18)$ & $0.0322(16)$ & $0.0162(15)$ & $0.0061(12)$ & $0.0073(13)$ \\
\hline $\mathrm{C} 7 \mathrm{~B}$ & $0.056(2)$ & $0.0468(18)$ & 0.0443 (19) & $0.0197(16)$ & $0.0020(15)$ & $-0.0011(14)$ \\
\hline $\mathrm{C} 8 \mathrm{~B}$ & $0.0414(18)$ & $0.0483(18)$ & $0.0370(17)$ & $0.0169(15)$ & $0.0093(14)$ & $0.0086(14)$ \\
\hline C9B & $0.0345(16)$ & $0.0461(17)$ & $0.0373(17)$ & $0.0137(14)$ & $0.0104(12)$ & $0.0083(13)$ \\
\hline C10B & $0.0464(18)$ & $0.0572(19)$ & $0.0385(17)$ & $0.0252(16)$ & $0.0092(14)$ & $0.0086(14)$ \\
\hline C11B & $0.0488(19)$ & $0.061(2)$ & $0.0369(18)$ & $0.0231(17)$ & $0.0097(14)$ & $0.0085(15)$ \\
\hline C12B & $0.0432(18)$ & $0.0501(18)$ & $0.0411(18)$ & $0.0109(15)$ & $0.0171(14)$ & $0.0046(14)$ \\
\hline C13B & $0.0414(18)$ & $0.059(2)$ & $0.053(2)$ & $0.0226(16)$ & $0.0099(15)$ & $0.0015(16)$ \\
\hline C14B & $0.0420(18)$ & $0.0561(19)$ & $0.0426(18)$ & $0.0192(16)$ & $0.0063(14)$ & $0.0067(15)$ \\
\hline C15B & $0.055(2)$ & $0.074(2)$ & $0.052(2)$ & 0.0217 (19) & $0.0174(16)$ & $-0.0008(17)$ \\
\hline $\mathrm{O} 1 \mathrm{~W}$ & $0.0640(15)$ & $0.0639(14)$ & $0.0506(13)$ & $0.0369(12)$ & $0.0237(11)$ & $0.0180(11)$ \\
\hline $\mathrm{O} 2 \mathrm{~W}$ & $0.0596(14)$ & $0.0589(14)$ & $0.0377(12)$ & $0.0211(12)$ & $0.0085(10)$ & $0.0093(10)$ \\
\hline $\mathrm{O} 3 \mathrm{~W}$ & $0.0558(14)$ & $0.0637(15)$ & $0.0429(12)$ & $0.0306(12)$ & $0.0092(10)$ & $0.0048(11)$ \\
\hline $\mathrm{O} 4 \mathrm{~W}$ & $0.0626(15)$ & 0.0585 (14) & $0.0422(12)$ & $0.0260(12)$ & $0.0129(10)$ & $0.0023(10)$ \\
\hline
\end{tabular}

Geometric parameters $\left(\AA,{ }^{\circ}\right)$

\begin{tabular}{llll}
\hline $\mathrm{Na} 1 \mathrm{~A}-\mathrm{Na}_{1} \mathrm{~A}^{\mathrm{i}}$ & $3.314(2)$ & $\mathrm{Na} 1 \mathrm{~B}-\mathrm{O} 1 \mathrm{~W}^{\mathrm{i}}$ & $2.607(3)$ \\
$\mathrm{Na} 1 \mathrm{~A}-\mathrm{O}_{1} \mathrm{~A}^{\mathrm{i}}$ & $2.441(2)$ & $\mathrm{Na} 1 \mathrm{~B}-\mathrm{O} 2 \mathrm{~W}^{\mathrm{iii}}$ & $2.538(2)$
\end{tabular}




\begin{tabular}{|c|c|c|c|}
\hline $\mathrm{Na} 1 \mathrm{~A}-\mathrm{O} 2 \mathrm{~A}$ & $2.298(2)$ & $\mathrm{Na} 1 \mathrm{~B}-\mathrm{O} 3 \mathrm{~W}$ & $2.417(2)$ \\
\hline $\mathrm{Na} 1 \mathrm{~A}-\mathrm{O} 2 \mathrm{~A}^{\mathrm{i}}$ & $2.636(2)$ & $\mathrm{Na} 1 \mathrm{~B}-\mathrm{O} 4 \mathrm{~W}$ & $2.460(2)$ \\
\hline $\mathrm{Na} 1 \mathrm{~A}-\mathrm{C} 1 \mathrm{~A}^{\mathrm{i}}$ & $2.838(3)$ & $\mathrm{O} 1 \mathrm{~B}-\mathrm{C} 1 \mathrm{~B}$ & $1.243(3)$ \\
\hline $\mathrm{Na} 1 \mathrm{~A}-\mathrm{Na} 1 \mathrm{~B}^{\mathrm{ii}}$ & $3.9976(16)$ & $\mathrm{O} 2 \mathrm{~B}-\mathrm{C} 1 \mathrm{~B}$ & $1.250(3)$ \\
\hline $\mathrm{Na} 1 \mathrm{~A}-\mathrm{Na} 1 \mathrm{~B}^{\mathrm{i}}$ & $3.2629(17)$ & $\mathrm{O} 3 \mathrm{~B}-\mathrm{C} 8 \mathrm{~B}$ & $1.241(4)$ \\
\hline $\mathrm{Na} 1 \mathrm{~A}-\mathrm{O} 1 \mathrm{~W}$ & $2.364(2)$ & $\mathrm{N} 1 \mathrm{~B}-\mathrm{C} 3 \mathrm{~B}$ & $1.362(4)$ \\
\hline $\mathrm{Na} 1 \mathrm{~A}-\mathrm{O} 2 \mathrm{~W}$ & $2.417(2)$ & $\mathrm{N} 1 \mathrm{~B}-\mathrm{C} 6 \mathrm{~B}$ & $1.385(4)$ \\
\hline $\mathrm{Na} 1 \mathrm{~A}-\mathrm{O} 4 \mathrm{~W}^{\mathrm{i}}$ & $2.493(2)$ & $\mathrm{N} 1 \mathrm{~B}-\mathrm{C} 7 \mathrm{~B}$ & $1.461(4)$ \\
\hline $\mathrm{O} 1 \mathrm{~A}-\mathrm{C} 1 \mathrm{~A}$ & $1.249(3)$ & $\mathrm{C} 1 \mathrm{~B}-\mathrm{C} 2 \mathrm{~B}$ & $1.522(4)$ \\
\hline $\mathrm{O} 1 \mathrm{~A}-\mathrm{Na} 1 \mathrm{~B}$ & $2.481(2)$ & $\mathrm{C} 2 \mathrm{~B}-\mathrm{H} 2 \mathrm{BA}$ & 0.9700 \\
\hline $\mathrm{O} 2 \mathrm{~A}-\mathrm{C} 1 \mathrm{~A}$ & $1.250(3)$ & $\mathrm{C} 2 \mathrm{~B}-\mathrm{H} 2 \mathrm{BB}$ & 0.9700 \\
\hline $\mathrm{O} 3 \mathrm{~A}-\mathrm{C} 8 \mathrm{~A}$ & $1.232(3)$ & $\mathrm{C} 2 \mathrm{~B}-\mathrm{C} 3 \mathrm{~B}$ & $1.479(4)$ \\
\hline $\mathrm{N} 1 \mathrm{~A}-\mathrm{C} 3 \mathrm{~A}$ & $1.359(4)$ & $\mathrm{C} 3 \mathrm{~B}-\mathrm{C} 4 \mathrm{~B}$ & $1.376(4)$ \\
\hline $\mathrm{N} 1 \mathrm{~A}-\mathrm{C} 6 \mathrm{~A}$ & 1.387 (3) & $\mathrm{C} 4 \mathrm{~B}-\mathrm{H} 4 \mathrm{~B}$ & 0.9300 \\
\hline N1A $-C 7 A$ & $1.461(4)$ & $\mathrm{C} 4 \mathrm{~B}-\mathrm{C} 5 \mathrm{~B}$ & $1.382(4)$ \\
\hline $\mathrm{C} 1 \mathrm{~A}-\mathrm{C} 2 \mathrm{~A}$ & $1.526(4)$ & $\mathrm{C} 5 \mathrm{~B}-\mathrm{H} 5 \mathrm{~B}$ & 0.9300 \\
\hline $\mathrm{C} 2 \mathrm{~A}-\mathrm{H} 2 \mathrm{AA}$ & 0.9700 & $\mathrm{C} 5 \mathrm{~B}-\mathrm{C} 6 \mathrm{~B}$ & $1.387(4)$ \\
\hline $\mathrm{C} 2 \mathrm{~A}-\mathrm{H} 2 \mathrm{AB}$ & 0.9700 & $\mathrm{C} 6 \mathrm{~B}-\mathrm{C} 8 \mathrm{~B}$ & $1.446(4)$ \\
\hline $\mathrm{C} 2 \mathrm{~A}-\mathrm{C} 3 \mathrm{~A}$ & $1.488(4)$ & $\mathrm{C} 7 \mathrm{~B}-\mathrm{H} 7 \mathrm{BA}$ & 0.9600 \\
\hline $\mathrm{C} 3 \mathrm{~A}-\mathrm{C} 4 \mathrm{~A}$ & $1.371(4)$ & $\mathrm{C} 7 \mathrm{~B}-\mathrm{H} 7 \mathrm{BB}$ & 0.9600 \\
\hline $\mathrm{C} 4 \mathrm{~A}-\mathrm{H} 4 \mathrm{~A}$ & 0.9300 & $\mathrm{C} 7 \mathrm{~B}-\mathrm{H} 7 \mathrm{BC}$ & 0.9600 \\
\hline $\mathrm{C} 4 \mathrm{~A}-\mathrm{C} 5 \mathrm{~A}$ & $1.384(4)$ & $\mathrm{C} 8 \mathrm{~B}-\mathrm{C} 9 \mathrm{~B}$ & $1.494(4)$ \\
\hline $\mathrm{C} 5 \mathrm{~A}-\mathrm{H} 5 \mathrm{~A}$ & 0.9300 & $\mathrm{C} 9 \mathrm{~B}-\mathrm{C} 10 \mathrm{~B}$ & $1.395(4)$ \\
\hline $\mathrm{C} 5 \mathrm{~A}-\mathrm{C} 6 \mathrm{~A}$ & $1.394(4)$ & $\mathrm{C} 9 \mathrm{~B}-\mathrm{C} 14 \mathrm{~B}$ & $1.371(4)$ \\
\hline $\mathrm{C} 6 \mathrm{~A}-\mathrm{C} 8 \mathrm{~A}$ & $1.448(4)$ & $\mathrm{C} 10 \mathrm{~B}-\mathrm{H} 10 \mathrm{~B}$ & 0.9300 \\
\hline $\mathrm{C} 7 \mathrm{~A}-\mathrm{H} 7 \mathrm{AA}$ & 0.9600 & $\mathrm{C} 10 \mathrm{~B}-\mathrm{C} 11 \mathrm{~B}$ & $1.376(4)$ \\
\hline $\mathrm{C} 7 \mathrm{~A}-\mathrm{H} 7 \mathrm{AB}$ & 0.9600 & C11B-H11B & 0.9300 \\
\hline $\mathrm{C} 7 \mathrm{~A}-\mathrm{H} 7 \mathrm{AC}$ & 0.9600 & $\mathrm{C} 11 \mathrm{~B}-\mathrm{C} 12 \mathrm{~B}$ & $1.393(4)$ \\
\hline $\mathrm{C} 8 \mathrm{~A}-\mathrm{C} 9 \mathrm{~A}$ & $1.494(4)$ & $\mathrm{C} 12 \mathrm{~B}-\mathrm{C} 13 \mathrm{~B}$ & $1.379(4)$ \\
\hline $\mathrm{C} 9 \mathrm{~A}-\mathrm{C} 10 \mathrm{~A}$ & $1.394(4)$ & $\mathrm{C} 12 \mathrm{~B}-\mathrm{C} 15 \mathrm{~B}$ & $1.510(4)$ \\
\hline $\mathrm{C} 9 \mathrm{~A}-\mathrm{C} 14 \mathrm{~A}$ & $1.387(4)$ & $\mathrm{C} 13 \mathrm{~B}-\mathrm{H} 13 \mathrm{~B}$ & 0.9300 \\
\hline $\mathrm{C} 10 \mathrm{~A}-\mathrm{H} 10 \mathrm{~A}$ & 0.9300 & $\mathrm{C} 13 \mathrm{~B}-\mathrm{C} 14 \mathrm{~B}$ & $1.380(4)$ \\
\hline $\mathrm{C} 10 \mathrm{~A}-\mathrm{C} 11 \mathrm{~A}$ & $1.376(4)$ & $\mathrm{C} 14 \mathrm{~B}-\mathrm{H} 14 \mathrm{~B}$ & 0.9300 \\
\hline $\mathrm{C} 11 \mathrm{~A}-\mathrm{H} 11 \mathrm{~A}$ & 0.9300 & $\mathrm{C} 15 \mathrm{~B}-\mathrm{H} 15 \mathrm{D}$ & 0.9600 \\
\hline $\mathrm{C} 11 \mathrm{~A}-\mathrm{C} 12 \mathrm{~A}$ & $1.391(4)$ & $\mathrm{C} 15 \mathrm{~B}-\mathrm{H} 15 \mathrm{E}$ & 0.9600 \\
\hline $\mathrm{C} 12 \mathrm{~A}-\mathrm{C} 13 \mathrm{~A}$ & $1.375(4)$ & $\mathrm{C} 15 \mathrm{~B}-\mathrm{H} 15 \mathrm{~F}$ & 0.9600 \\
\hline $\mathrm{C} 12 \mathrm{~A}-\mathrm{C} 15 \mathrm{~A}$ & $1.503(4)$ & $\mathrm{O} 1 \mathrm{~W}-\mathrm{H} 1 \mathrm{WA}$ & 0.8897 \\
\hline $\mathrm{C} 13 \mathrm{~A}-\mathrm{H} 13 \mathrm{~A}$ & 0.9300 & $\mathrm{O} 1 \mathrm{~W}-\mathrm{H} 1 \mathrm{WB}$ & 0.8899 \\
\hline $\mathrm{C} 13 \mathrm{~A}-\mathrm{C} 14 \mathrm{~A}$ & $1.384(4)$ & $\mathrm{O} 2 \mathrm{~W}-\mathrm{H} 2 \mathrm{WA}$ & 0.8899 \\
\hline $\mathrm{C} 14 \mathrm{~A}-\mathrm{H} 14 \mathrm{~A}$ & 0.9300 & $\mathrm{O} 2 \mathrm{~W}-\mathrm{H} 2 \mathrm{WB}$ & 0.8899 \\
\hline $\mathrm{C} 15 \mathrm{~A}-\mathrm{H} 15 \mathrm{~A}$ & 0.9600 & $\mathrm{O} 3 \mathrm{~W}-\mathrm{H} 3 \mathrm{WA}$ & 0.8905 \\
\hline $\mathrm{C} 15 \mathrm{~A}-\mathrm{H} 15 \mathrm{~B}$ & 0.9600 & $\mathrm{O} 3 \mathrm{~W}-\mathrm{H} 3 \mathrm{WB}$ & 0.8905 \\
\hline $\mathrm{C} 15 \mathrm{~A}-\mathrm{H} 15 \mathrm{C}$ & 0.9600 & $\mathrm{O} 4 \mathrm{~W}-\mathrm{H} 4 \mathrm{WA}$ & 0.8897 \\
\hline $\mathrm{Na} 1 \mathrm{~B}-\mathrm{O} 1 \mathrm{~B}$ & $2.416(2)$ & $\mathrm{O} 4 \mathrm{~W}-\mathrm{H} 4 \mathrm{WB}$ & 0.8902 \\
\hline $\mathrm{Na} 1 \mathrm{~A}-\mathrm{Na} 1 \mathrm{~A}-\mathrm{Na} 1 \mathrm{~B}^{\mathrm{ii}}$ & $135.58(5)$ & $\mathrm{H} 15 \mathrm{~A}-\mathrm{C} 15 \mathrm{~A}-\mathrm{H} 15 \mathrm{C}$ & 109.5 \\
\hline $\mathrm{O} 1 \mathrm{~A}-\mathrm{Na} 1 \mathrm{~A}-\mathrm{Na} 1 \mathrm{~A}^{\mathrm{i}}$ & $94.89(7)$ & $\mathrm{H} 15 \mathrm{~B}-\mathrm{C} 15 \mathrm{~A}-\mathrm{H} 15 \mathrm{C}$ & 109.5 \\
\hline $\mathrm{O} 1 \mathrm{~A}^{\mathrm{i}}-\mathrm{Na} 1 \mathrm{~A}-\mathrm{O} 2 \mathrm{~A}^{\mathrm{i}}$ & $51.31(6)$ & $\mathrm{Na} 1 \mathrm{~A}-\mathrm{Na} 1 \mathrm{~B}-\mathrm{Na} 1 \mathrm{~A}^{\mathrm{iii}}$ & $103.67(4)$ \\
\hline
\end{tabular}




\begin{tabular}{|c|c|}
\hline $\mathrm{O} 1 \mathrm{~A}-\mathrm{Na} 1 \mathrm{~A}-\mathrm{C} 1 \mathrm{~A}^{\mathrm{i}}$ & $26.00(7)$ \\
\hline $\mathrm{O} 1 \mathrm{~A}^{\mathrm{i}}-\mathrm{Na} 1 \mathrm{~A}-\mathrm{Na} 1 \mathrm{~B}^{\mathrm{i}}$ & $49.01(5)$ \\
\hline $\mathrm{O} 1 \mathrm{~A}^{\mathrm{i}}-\mathrm{Na} 1 \mathrm{~A}-\mathrm{Na} 1 \mathrm{~B}^{\mathrm{ii}}$ & $61.96(6)$ \\
\hline $\mathrm{O} 1 \mathrm{~A}-\mathrm{Na} 1 \mathrm{~A}-\mathrm{O} 4 \mathrm{~W}^{\mathrm{i}}$ & $78.93(8)$ \\
\hline $\mathrm{O} 2 \mathrm{~A}^{\mathrm{i}}-\mathrm{Na} 1 \mathrm{~A}-\mathrm{Na} 1 \mathrm{~A}^{\mathrm{i}}$ & $43.62(5)$ \\
\hline $\mathrm{O} 2 \mathrm{~A}-\mathrm{Na} 1 \mathrm{~A}-\mathrm{Na} 1 \mathrm{~A}^{\mathrm{i}}$ & $52.29(6)$ \\
\hline $\mathrm{O} 2 \mathrm{~A}-\mathrm{Na} 1 \mathrm{~A}-\mathrm{O} 1 \mathrm{~A}^{\mathrm{i}}$ & $147.11(9)$ \\
\hline $\mathrm{O} 2 \mathrm{~A}-\mathrm{Na} 1 \mathrm{~A}-\mathrm{O} 2 \mathrm{~A}^{\mathrm{i}}$ & $95.91(8)$ \\
\hline $\mathrm{O} 2 \mathrm{~A}^{\mathrm{i}}-\mathrm{Na} 1 \mathrm{~A}-\mathrm{C} 1 \mathrm{~A}^{\mathrm{i}}$ & $26.07(7)$ \\
\hline $\mathrm{O} 2 \mathrm{~A}-\mathrm{Na} 1 \mathrm{~A}-\mathrm{C} 1 \mathrm{~A}^{\mathrm{i}}$ & $121.17(9)$ \\
\hline $\mathrm{O} 2 \mathrm{~A}^{\mathrm{i}}-\mathrm{Na} 1 \mathrm{~A}-\mathrm{Na} 1 \mathrm{~B}^{\mathrm{ii}}$ & $104.45(6)$ \\
\hline $\mathrm{O} 2 \mathrm{~A}-\mathrm{Na} 1 \mathrm{~A}-\mathrm{Na} 1 \mathrm{~B}^{\mathrm{i}}$ & $142.94(8)$ \\
\hline $\mathrm{O} 2 \mathrm{~A}^{\mathrm{i}}-\mathrm{Na} 1 \mathrm{~A}-\mathrm{Na} 1 \mathrm{~B}^{\mathrm{i}}$ & $84.23(6)$ \\
\hline $\mathrm{O} 2 \mathrm{~A}-\mathrm{Na} 1 \mathrm{~A}-\mathrm{Na} 1 \mathrm{~B}^{\mathrm{ii}}$ & $138.04(8)$ \\
\hline $\mathrm{O} 2 \mathrm{~A}-\mathrm{Na} 1 \mathrm{~A}-\mathrm{O} 1 \mathrm{~W}$ & $121.00(9)$ \\
\hline $\mathrm{O} 2 \mathrm{~A}-\mathrm{Na} 1 \mathrm{~A}-\mathrm{O} 2 \mathrm{~W}$ & $103.90(9)$ \\
\hline $\mathrm{O} 2 \mathrm{~A}-\mathrm{Na} 1 \mathrm{~A}-\mathrm{O} 4 \mathrm{~W}^{\mathrm{i}}$ & $95.91(8)$ \\
\hline $\mathrm{C} 1 \mathrm{~A}^{\mathrm{i}}-\mathrm{Na} 1 \mathrm{~A}-\mathrm{Na} 1 \mathrm{~A}^{\mathrm{i}}$ & $69.18(7)$ \\
\hline $\mathrm{C} 1 \mathrm{~A}^{\mathrm{i}}-\mathrm{Na} 1 \mathrm{~A}-\mathrm{Na} 1 \mathrm{~B}^{\mathrm{ii}}$ & $80.46(6)$ \\
\hline 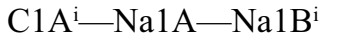 & $68.45(6)$ \\
\hline $\mathrm{Na} 1 \mathrm{~B}-\mathrm{Na} 1 \mathrm{~A}-\mathrm{Na} 1 \mathrm{~A}^{\mathrm{i}}$ & $118.26(6)$ \\
\hline $\mathrm{Na} 1 \mathrm{~B}{ }^{\mathrm{i}}-\mathrm{Na} 1 \mathrm{~A}-\mathrm{Na} 1 \mathrm{~B}^{\mathrm{ii}}$ & $76.33(4)$ \\
\hline $\mathrm{O} 1 \mathrm{~W}-\mathrm{Na} 1 \mathrm{~A}-\mathrm{Na} 1 \mathrm{~A}^{\mathrm{i}}$ & $158.68(9)$ \\
\hline $\mathrm{O} 1 \mathrm{~W}-\mathrm{Na} 1 \mathrm{~A}-\mathrm{O} 1 \mathrm{~A}$ & $90.21(8)$ \\
\hline $\mathrm{O} 1 \mathrm{~W}-\mathrm{Na} 1 \mathrm{~A}-\mathrm{O} 2 \mathrm{~A}^{\mathrm{i}}$ & $136.23(9)$ \\
\hline $\mathrm{O} 1 \mathrm{~W}-\mathrm{Na} 1 \mathrm{~A}-\mathrm{C} 1 \mathrm{~A}^{\mathrm{i}}$ & $115.58(9)$ \\
\hline $\mathrm{O} 1 \mathrm{~W}-\mathrm{Na} 1 \mathrm{~A}-\mathrm{Na} 1 \mathrm{~B}^{\mathrm{i}}$ & $52.29(6)$ \\
\hline $\mathrm{O} 1 \mathrm{~W}-\mathrm{Na} 1 \mathrm{~A}-\mathrm{Na} 1 \mathrm{~B}^{\mathrm{ii}}$ & $64.50(6)$ \\
\hline $\mathrm{O} 1 \mathrm{~W}-\mathrm{Na} 1 \mathrm{~A}-\mathrm{O} 2 \mathrm{~W}$ & $93.84(8)$ \\
\hline $\mathrm{O} 1 \mathrm{~W}-\mathrm{Na} 1 \mathrm{~A}-\mathrm{O} 4 \mathrm{~W}^{\mathrm{i}}$ & $79.14(8)$ \\
\hline $\mathrm{O} 2 \mathrm{~W}-\mathrm{Na} 1 \mathrm{~A}-\mathrm{Na} 1 \mathrm{~A}^{\mathrm{i}}$ & $107.35(7)$ \\
\hline $\mathrm{O} 2 \mathrm{~W}-\mathrm{Na} 1 \mathrm{~A}-\mathrm{O} 1 \mathrm{~A}^{\mathrm{i}}$ & $82.17(8)$ \\
\hline $\mathrm{O} 2 \mathrm{~W}-\mathrm{Na} 1 \mathrm{~A}-\mathrm{O} 2 \mathrm{~A}^{\mathrm{i}}$ & $99.52(8)$ \\
\hline $\mathrm{O} 2 \mathrm{~W}-\mathrm{Na} 1 \mathrm{~A}-\mathrm{C} 1 \mathrm{~A}^{\mathrm{i}}$ & $86.62(8)$ \\
\hline $\mathrm{O} 2 \mathrm{~W}-\mathrm{Na} 1 \mathrm{~A}-\mathrm{Na} 1 \mathrm{~B}^{\mathrm{i}}$ & $112.65(7)$ \\
\hline $\mathrm{O} 2 \mathrm{~W}-\mathrm{Na} 1 \mathrm{~A}-\mathrm{Na} 1 \mathrm{~B}^{\mathrm{ii}}$ & $37.26(6)$ \\
\hline $\mathrm{O} 2 \mathrm{~W}-\mathrm{Na} 1 \mathrm{~A}-\mathrm{O} 4 \mathrm{~W}^{\mathrm{i}}$ & $159.77(8)$ \\
\hline $\mathrm{O} 4 \mathrm{~W}^{\mathrm{i}}-\mathrm{Na} 1 \mathrm{~A}-\mathrm{Na} 1 \mathrm{~A}^{\mathrm{i}}$ & $81.55(7)$ \\
\hline $\mathrm{O} 4 \mathrm{~W}^{\mathrm{i}}-\mathrm{Na} 1 \mathrm{~A}-\mathrm{O} 2 \mathrm{~A}^{\mathrm{i}}$ & $74.06(8)$ \\
\hline $\mathrm{O} 4 \mathrm{~W}^{\mathrm{i}}-\mathrm{Na} 1 \mathrm{~A}-\mathrm{C} 1 \mathrm{~A}^{\mathrm{i}}$ & $79.57(8)$ \\
\hline $\mathrm{O} 4 \mathrm{~W}^{\mathrm{i}}-\mathrm{Na} 1 \mathrm{~A}-\mathrm{Na} 1 \mathrm{~B}^{\mathrm{i}}$ & $48.35(6)$ \\
\hline $\mathrm{O} 4 \mathrm{~W}^{\mathrm{i}}-\mathrm{Na} 1 \mathrm{~A}-\mathrm{Na} 1 \mathrm{~B}^{\mathrm{ii}}$ & $124.68(6)$ \\
\hline $\mathrm{Na} 1 \mathrm{~A}-\mathrm{O} 1 \mathrm{~A}-\mathrm{Na} 1 \mathrm{~B}$ & $83.04(7)$ \\
\hline $\mathrm{C} 1 \mathrm{~A}-\mathrm{O} 1 \mathrm{~A}-\mathrm{Na} 1 \mathrm{~A}^{\mathrm{i}}$ & $95.05(17)$ \\
\hline $\mathrm{C} 1 \mathrm{~A}-\mathrm{O} 1 \mathrm{~A}-\mathrm{Na} 1 \mathrm{~B}$ & $132.45(19$ \\
\hline $\mathrm{Na} 1 \mathrm{~A}-\mathrm{O} 2 \mathrm{~A}-\mathrm{Na} 1 \mathrm{~A}^{\mathrm{i}}$ & $84.09(8)$ \\
\hline $\mathrm{C} 1 \mathrm{~A}-\mathrm{O} 2 \mathrm{~A}-\mathrm{Na} 1 \mathrm{~A}^{\mathrm{i}}$ & $86.05(17)$ \\
\hline $\mathrm{C} 1 \mathrm{~A}-\mathrm{O} 2 \mathrm{~A}-\mathrm{Na} 1 \mathrm{~A}$ & $163.4(2)$ \\
\hline
\end{tabular}

\begin{tabular}{|c|c|}
\hline $\mathrm{O} 1 \mathrm{~A}-\mathrm{Na} 1 \mathrm{~B}-\mathrm{Na} 1 \mathrm{~A}^{\mathrm{i}}$ & $47.95(5)$ \\
\hline $\mathrm{O} 1 \mathrm{~A}-\mathrm{Na} 1 \mathrm{~B}-\mathrm{Na} 1 \mathrm{~A}^{\mathrm{iii}}$ & $81.27(6)$ \\
\hline $\mathrm{O} 1 \mathrm{~A}-\mathrm{Na} 1 \mathrm{~B}-\mathrm{O} 1 \mathrm{~W}^{\mathrm{i}}$ & $83.94(8)$ \\
\hline $\mathrm{O} 1 \mathrm{~A}-\mathrm{Na} 1 \mathrm{~B}-\mathrm{O} 2 \mathrm{~W}^{\mathrm{iii}}$ & $111.77(8)$ \\
\hline $\mathrm{O} 1 \mathrm{~B}-\mathrm{Na} 1 \mathrm{~B}-\mathrm{Na} 1 \mathrm{~A}^{\mathrm{i}}$ & $116.87(7)$ \\
\hline $\mathrm{O} 1 \mathrm{~B}-\mathrm{Na} 1 \mathrm{~B}-\mathrm{Na} 1 \mathrm{~A}^{\mathrm{iii}}$ & $107.75(6)$ \\
\hline $\mathrm{O} 1 \mathrm{~B}-\mathrm{Na} 1 \mathrm{~B}-\mathrm{O} 1 \mathrm{~A}$ & $164.64(8)$ \\
\hline $\mathrm{O} 1 \mathrm{~B}-\mathrm{Na} 1 \mathrm{~B}-\mathrm{O} 1 \mathrm{~W}^{\mathrm{i}}$ & $84.57(8)$ \\
\hline $\mathrm{O} 1 \mathrm{~B}-\mathrm{Na} 1 \mathrm{~B}-\mathrm{O} 2 \mathrm{~W}^{\mathrm{iii}}$ & $81.05(7)$ \\
\hline $\mathrm{O} 1 \mathrm{~B}-\mathrm{Na} 1 \mathrm{~B}-\mathrm{O} 3 \mathrm{~W}$ & $101.75(9)$ \\
\hline $\mathrm{O} 1 \mathrm{~B}-\mathrm{Na} 1 \mathrm{~B}-\mathrm{O} 4 \mathrm{~W}$ & $88.42(8)$ \\
\hline 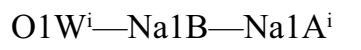 & $45.82(5)$ \\
\hline $\mathrm{O} 1 \mathrm{~W}^{\mathrm{i}}-\mathrm{Na} 1 \mathrm{~B}-\mathrm{Na} 1 \mathrm{~A}^{\mathrm{iii}}$ & $84.57(6)$ \\
\hline $\mathrm{O} 2 \mathrm{~W}^{\mathrm{iii}}-\mathrm{Na} 1 \mathrm{~B}-\mathrm{Na} 1 \mathrm{~A}^{\mathrm{iii}}$ & $35.20(5)$ \\
\hline $\mathrm{O} 2 \mathrm{~W}^{\mathrm{iii}}-\mathrm{Na} 1 \mathrm{~B}-\mathrm{Na} 1 \mathrm{~A}^{\mathrm{i}}$ & $137.63(7)$ \\
\hline $\mathrm{O} 2 \mathrm{~W}^{\mathrm{iii}}-\mathrm{Na} 1 \mathrm{~B}-\mathrm{O} 1 \mathrm{~W}^{\mathrm{i}}$ & $104.60(8)$ \\
\hline $\mathrm{O} 3 \mathrm{~W}-\mathrm{Na} 1 \mathrm{~B}-\mathrm{Na} 1 \mathrm{~A}^{\mathrm{iii}}$ & $107.78(6)$ \\
\hline $\mathrm{O} 3 \mathrm{~W}-\mathrm{Na} 1 \mathrm{~B}-\mathrm{Na} 1 \mathrm{~A}^{\mathrm{i}}$ & $118.49(7)$ \\
\hline $\mathrm{O} 3 \mathrm{~W}-\mathrm{Na} 1 \mathrm{~B}-\mathrm{O} 1 \mathrm{~A}$ & $86.73(8)$ \\
\hline $\mathrm{O} 3 \mathrm{~W}-\mathrm{Na} 1 \mathrm{~B}-\mathrm{O} 1 \mathrm{~W}^{\mathrm{i}}$ & $163.22(8)$ \\
\hline $\mathrm{O} 3 \mathrm{~W}-\mathrm{Na} 1 \mathrm{~B}-\mathrm{O} 2 \mathrm{~W}^{\mathrm{iii}}$ & $91.80(8)$ \\
\hline $\mathrm{O} 3 \mathrm{~W}-\mathrm{Na} 1 \mathrm{~B}-\mathrm{O} 4 \mathrm{~W}$ & $89.29(8)$ \\
\hline $\mathrm{O} 4 \mathrm{~W}-\mathrm{Na} 1 \mathrm{~B}-\mathrm{Na} 1 \mathrm{~A}^{\mathrm{iii}}$ & $152.90(7)$ \\
\hline $\mathrm{O} 4 \mathrm{~W}-\mathrm{Na} 1 \mathrm{~B}-\mathrm{Na} 1 \mathrm{~A}^{\mathrm{i}}$ & $49.23(6)$ \\
\hline $\mathrm{O} 4 \mathrm{~W}-\mathrm{Na} 1 \mathrm{~B}-\mathrm{O} 1 \mathrm{~A}$ & $78.81(8)$ \\
\hline $\mathrm{O} 4 \mathrm{~W}-\mathrm{Na} 1 \mathrm{~B}-\mathrm{O} 1 \mathrm{~W}^{\mathrm{i}}$ & $75.24(8)$ \\
\hline $\mathrm{O} 4 \mathrm{~W}-\mathrm{Na} 1 \mathrm{~B}-\mathrm{O} 2 \mathrm{~W}^{\mathrm{iii}}$ & $169.41(8)$ \\
\hline $\mathrm{C} 1 \mathrm{~B}-\mathrm{O} 1 \mathrm{~B}-\mathrm{Na} 1 \mathrm{~B}$ & $126.86(19)$ \\
\hline $\mathrm{C} 3 \mathrm{~B}-\mathrm{N} 1 \mathrm{~B}-\mathrm{C} 6 \mathrm{~B}$ & $109.0(2)$ \\
\hline $\mathrm{C} 3 \mathrm{~B}-\mathrm{N} 1 \mathrm{~B}-\mathrm{C} 7 \mathrm{~B}$ & $125.0(2)$ \\
\hline $\mathrm{C} 6 \mathrm{~B}-\mathrm{N} 1 \mathrm{~B}-\mathrm{C} 7 \mathrm{~B}$ & $125.7(2)$ \\
\hline $\mathrm{O} 1 \mathrm{~B}-\mathrm{C} 1 \mathrm{~B}-\mathrm{O} 2 \mathrm{~B}$ & $125.0(3)$ \\
\hline $\mathrm{O} 1 \mathrm{~B}-\mathrm{C} 1 \mathrm{~B}-\mathrm{C} 2 \mathrm{~B}$ & $116.3(3)$ \\
\hline $\mathrm{O} 2 \mathrm{~B}-\mathrm{C} 1 \mathrm{~B}-\mathrm{C} 2 \mathrm{~B}$ & $118.7(2)$ \\
\hline $\mathrm{C} 1 \mathrm{~B}-\mathrm{C} 2 \mathrm{~B}-\mathrm{H} 2 \mathrm{BA}$ & 108.2 \\
\hline $\mathrm{C} 1 \mathrm{~B}-\mathrm{C} 2 \mathrm{~B}-\mathrm{H} 2 \mathrm{BB}$ & 108.2 \\
\hline $\mathrm{H} 2 \mathrm{BA}-\mathrm{C} 2 \mathrm{~B}-\mathrm{H} 2 \mathrm{BB}$ & 107.3 \\
\hline $\mathrm{C} 3 \mathrm{~B}-\mathrm{C} 2 \mathrm{~B}-\mathrm{C} 1 \mathrm{~B}$ & $116.5(2)$ \\
\hline $\mathrm{C} 3 \mathrm{~B}-\mathrm{C} 2 \mathrm{~B}-\mathrm{H} 2 \mathrm{BA}$ & 108.2 \\
\hline $\mathrm{C} 3 \mathrm{~B}-\mathrm{C} 2 \mathrm{~B}-\mathrm{H} 2 \mathrm{BB}$ & 108.2 \\
\hline $\mathrm{N} 1 \mathrm{~B}-\mathrm{C} 3 \mathrm{~B}-\mathrm{C} 2 \mathrm{~B}$ & $123.9(3)$ \\
\hline $\mathrm{N} 1 \mathrm{~B}-\mathrm{C} 3 \mathrm{~B}-\mathrm{C} 4 \mathrm{~B}$ & $107.9(2)$ \\
\hline $\mathrm{C} 4 \mathrm{~B}-\mathrm{C} 3 \mathrm{~B}-\mathrm{C} 2 \mathrm{~B}$ & $128.1(3)$ \\
\hline $\mathrm{C} 3 \mathrm{~B}-\mathrm{C} 4 \mathrm{~B}-\mathrm{H} 4 \mathrm{~B}$ & 125.9 \\
\hline $\mathrm{C} 3 \mathrm{~B}-\mathrm{C} 4 \mathrm{~B}-\mathrm{C} 5 \mathrm{~B}$ & $108.2(3)$ \\
\hline $\mathrm{C} 5 \mathrm{~B}-\mathrm{C} 4 \mathrm{~B}-\mathrm{H} 4 \mathrm{~B}$ & 125.9 \\
\hline $\mathrm{C} 4 \mathrm{~B}-\mathrm{C} 5 \mathrm{~B}-\mathrm{H} 5 \mathrm{~B}$ & 126.1 \\
\hline $\mathrm{C} 4 \mathrm{~B}-\mathrm{C} 5 \mathrm{~B}-\mathrm{C} 6 \mathrm{~B}$ & $107.8(3)$ \\
\hline
\end{tabular}




\begin{tabular}{|c|c|}
\hline $\mathrm{C} 3 \mathrm{~A}-\mathrm{N} 1 \mathrm{~A}-\mathrm{C} 6 \mathrm{~A}$ & $109.3(2)$ \\
\hline $\mathrm{C} 3 \mathrm{~A}-\mathrm{N} 1 \mathrm{~A}-\mathrm{C} 7 \mathrm{~A}$ & $124.8(2)$ \\
\hline $\mathrm{C} 6 \mathrm{~A}-\mathrm{N} 1 \mathrm{~A}-\mathrm{C} 7 \mathrm{~A}$ & $125.4(2)$ \\
\hline $\mathrm{O} 1 \mathrm{~A}-\mathrm{C} 1 \mathrm{~A}-\mathrm{Na} 1 \mathrm{~A}^{\mathrm{i}}$ & $58.95(14)$ \\
\hline $\mathrm{O} 1 \mathrm{~A}-\mathrm{C} 1 \mathrm{~A}-\mathrm{O} 2 \mathrm{~A}$ & $123.8(3)$ \\
\hline $\mathrm{O} 1 \mathrm{~A}-\mathrm{C} 1 \mathrm{~A}-\mathrm{C} 2 \mathrm{~A}$ & $116.3(2)$ \\
\hline $\mathrm{O} 2 \mathrm{~A}-\mathrm{C} 1 \mathrm{~A}-\mathrm{Na} 1 \mathrm{~A}^{\mathrm{i}}$ & $67.89(15)$ \\
\hline $\mathrm{O} 2 \mathrm{~A}-\mathrm{C} 1 \mathrm{~A}-\mathrm{C} 2 \mathrm{~A}$ & $119.8(2)$ \\
\hline $\mathrm{C} 2 \mathrm{~A}-\mathrm{C} 1 \mathrm{~A}-\mathrm{Na} 1 \mathrm{~A}^{\mathrm{i}}$ & $158.3(2)$ \\
\hline $\mathrm{C} 1 \mathrm{~A}-\mathrm{C} 2 \mathrm{~A}-\mathrm{H} 2 \mathrm{AA}$ & 107.9 \\
\hline $\mathrm{C} 1 \mathrm{~A}-\mathrm{C} 2 \mathrm{~A}-\mathrm{H} 2 \mathrm{AB}$ & 107.9 \\
\hline $\mathrm{H} 2 \mathrm{AA}-\mathrm{C} 2 \mathrm{~A}-\mathrm{H} 2 \mathrm{AB}$ & 107.2 \\
\hline $\mathrm{C} 3 \mathrm{~A}-\mathrm{C} 2 \mathrm{~A}-\mathrm{C} 1 \mathrm{~A}$ & $117.4(2)$ \\
\hline $\mathrm{C} 3 \mathrm{~A}-\mathrm{C} 2 \mathrm{~A}-\mathrm{H} 2 \mathrm{AA}$ & 107.9 \\
\hline $\mathrm{C} 3 \mathrm{~A}-\mathrm{C} 2 \mathrm{~A}-\mathrm{H} 2 \mathrm{AB}$ & 107.9 \\
\hline $\mathrm{N} 1 \mathrm{~A}-\mathrm{C} 3 \mathrm{~A}-\mathrm{C} 2 \mathrm{~A}$ & $123.8(3)$ \\
\hline $\mathrm{N} 1 \mathrm{~A}-\mathrm{C} 3 \mathrm{~A}-\mathrm{C} 4 \mathrm{~A}$ & $108.1(2)$ \\
\hline $\mathrm{C} 4 \mathrm{~A}-\mathrm{C} 3 \mathrm{~A}-\mathrm{C} 2 \mathrm{~A}$ & $128.1(3)$ \\
\hline $\mathrm{C} 3 \mathrm{~A}-\mathrm{C} 4 \mathrm{~A}-\mathrm{H} 4 \mathrm{~A}$ & 125.8 \\
\hline $\mathrm{C} 3 \mathrm{~A}-\mathrm{C} 4 \mathrm{~A}-\mathrm{C} 5 \mathrm{~A}$ & $108.4(3)$ \\
\hline $\mathrm{C} 5 \mathrm{~A}-\mathrm{C} 4 \mathrm{~A}-\mathrm{H} 4 \mathrm{~A}$ & 125.8 \\
\hline $\mathrm{C} 4 \mathrm{~A}-\mathrm{C} 5 \mathrm{~A}-\mathrm{H} 5 \mathrm{~A}$ & 126.2 \\
\hline $\mathrm{C} 4 \mathrm{~A}-\mathrm{C} 5 \mathrm{~A}-\mathrm{C} 6 \mathrm{~A}$ & $107.6(3)$ \\
\hline $\mathrm{C} 6 \mathrm{~A}-\mathrm{C} 5 \mathrm{~A}-\mathrm{H} 5 \mathrm{~A}$ & 126.2 \\
\hline $\mathrm{N} 1 \mathrm{~A}-\mathrm{C} 6 \mathrm{~A}-\mathrm{C} 5 \mathrm{~A}$ & $106.6(3)$ \\
\hline $\mathrm{N} 1 \mathrm{~A}-\mathrm{C} 6 \mathrm{~A}-\mathrm{C} 8 \mathrm{~A}$ & $124.4(3)$ \\
\hline $\mathrm{C} 5 \mathrm{~A}-\mathrm{C} 6 \mathrm{~A}-\mathrm{C} 8 \mathrm{~A}$ & $127.6(3)$ \\
\hline $\mathrm{N} 1 \mathrm{~A}-\mathrm{C} 7 \mathrm{~A}-\mathrm{H} 7 \mathrm{AA}$ & 109.5 \\
\hline $\mathrm{N} 1 \mathrm{~A}-\mathrm{C} 7 \mathrm{~A}-\mathrm{H} 7 \mathrm{AB}$ & 109.5 \\
\hline $\mathrm{N} 1 \mathrm{~A}-\mathrm{C} 7 \mathrm{~A}-\mathrm{H} 7 \mathrm{AC}$ & 109.5 \\
\hline H7AA $-\mathrm{C} 7 \mathrm{~A}-\mathrm{H} 7 \mathrm{AB}$ & 109.5 \\
\hline $\mathrm{H} 7 \mathrm{AA}-\mathrm{C} 7 \mathrm{~A}-\mathrm{H} 7 \mathrm{AC}$ & 109.5 \\
\hline $\mathrm{H} 7 \mathrm{AB}-\mathrm{C} 7 \mathrm{~A}-\mathrm{H} 7 \mathrm{AC}$ & 109.5 \\
\hline $\mathrm{O} 3 \mathrm{~A}-\mathrm{C} 8 \mathrm{~A}-\mathrm{C} 6 \mathrm{~A}$ & 123.4 \\
\hline $\mathrm{O} 3 \mathrm{~A}-\mathrm{C} 8 \mathrm{~A}-\mathrm{C} 9 \mathrm{~A}$ & $119.5(3)$ \\
\hline $\mathrm{C} 6 \mathrm{~A}-\mathrm{C} 8 \mathrm{~A}-\mathrm{C} 9 \mathrm{~A}$ & $117.0(3)$ \\
\hline $\mathrm{C} 10 \mathrm{~A}-\mathrm{C} 9 \mathrm{~A}-\mathrm{C} 8 \mathrm{~A}$ & $122.0(3)$ \\
\hline $\mathrm{C} 14 \mathrm{~A}-\mathrm{C} 9 \mathrm{~A}-\mathrm{C} 8 \mathrm{~A}$ & $119.9(3)$ \\
\hline $\mathrm{C} 14 \mathrm{~A}-\mathrm{C} 9 \mathrm{~A}-\mathrm{C} 10 \mathrm{~A}$ & $118.0(3)$ \\
\hline $\mathrm{C} 9 \mathrm{~A}-\mathrm{C} 10 \mathrm{~A}-\mathrm{H} 10 \mathrm{~A}$ & 119.7 \\
\hline $\mathrm{C} 11 \mathrm{~A}-\mathrm{C} 10 \mathrm{~A}-\mathrm{C} 9 \mathrm{~A}$ & 120.5 \\
\hline $\mathrm{C} 11 \mathrm{~A}-\mathrm{C} 10 \mathrm{~A}-\mathrm{H} 10 \mathrm{~A}$ & 119.7 \\
\hline $\mathrm{C} 10 \mathrm{~A}-\mathrm{C} 11 \mathrm{~A}-\mathrm{H} 11 \mathrm{~A}$ & 119.2 \\
\hline $\mathrm{C} 10 \mathrm{~A}-\mathrm{C} 11 \mathrm{~A}-\mathrm{C} 12 \mathrm{~A}$ & $121.5(3)$ \\
\hline $\mathrm{C} 12 \mathrm{~A}-\mathrm{C} 11 \mathrm{~A}-\mathrm{H} 11 \mathrm{~A}$ & 119.2 \\
\hline $\mathrm{C} 11 \mathrm{~A}-\mathrm{C} 12 \mathrm{~A}-\mathrm{C} 15 \mathrm{~A}$ & $119.7(3)$ \\
\hline $\mathrm{C} 13 \mathrm{~A}-\mathrm{C} 12 \mathrm{~A}-\mathrm{C} 11 \mathrm{~A}$ & $117.6(3)$ \\
\hline $\mathrm{C} 13 \mathrm{~A}-\mathrm{C} 12 \mathrm{~A}-\mathrm{C} 15 \mathrm{~A}$ & $122.7(3)$ \\
\hline
\end{tabular}

\begin{tabular}{|c|c|}
\hline $\mathrm{C} 6 \mathrm{~B}-\mathrm{C} 5 \mathrm{~B}-\mathrm{H} 5 \mathrm{~B}$ & 126.1 \\
\hline $\mathrm{N} 1 \mathrm{~B}-\mathrm{C} 6 \mathrm{~B}-\mathrm{C} 5 \mathrm{~B}$ & $107.0(3)$ \\
\hline $\mathrm{N} 1 \mathrm{~B}-\mathrm{C} 6 \mathrm{~B}-\mathrm{C} 8 \mathrm{~B}$ & $123.9(3)$ \\
\hline $\mathrm{C} 5 \mathrm{~B}-\mathrm{C} 6 \mathrm{~B}-\mathrm{C} 8 \mathrm{~B}$ & $127.8(3)$ \\
\hline $\mathrm{N} 1 \mathrm{~B}-\mathrm{C} 7 \mathrm{~B}-\mathrm{H} 7 \mathrm{BA}$ & 109.5 \\
\hline $\mathrm{N} 1 \mathrm{~B}-\mathrm{C} 7 \mathrm{~B}-\mathrm{H} 7 \mathrm{BB}$ & 109.5 \\
\hline $\mathrm{N} 1 \mathrm{~B}-\mathrm{C} 7 \mathrm{~B}-\mathrm{H} 7 \mathrm{BC}$ & 109.5 \\
\hline $\mathrm{H} 7 \mathrm{BA}-\mathrm{C} 7 \mathrm{~B}-\mathrm{H} 7 \mathrm{BB}$ & 109.5 \\
\hline $\mathrm{H} 7 \mathrm{BA}-\mathrm{C} 7 \mathrm{~B}-\mathrm{H} 7 \mathrm{BC}$ & 109.5 \\
\hline $\mathrm{H} 7 \mathrm{BB}-\mathrm{C} 7 \mathrm{~B}-\mathrm{H} 7 \mathrm{BC}$ & 109.5 \\
\hline $\mathrm{O} 3 \mathrm{~B}-\mathrm{C} 8 \mathrm{~B}-\mathrm{C} 6 \mathrm{~B}$ & $123.2(3)$ \\
\hline $\mathrm{O} 3 \mathrm{~B}-\mathrm{C} 8 \mathrm{~B}-\mathrm{C} 9 \mathrm{~B}$ & $119.5(3)$ \\
\hline $\mathrm{C} 6 \mathrm{~B}-\mathrm{C} 8 \mathrm{~B}-\mathrm{C} 9 \mathrm{~B}$ & $117.3(3)$ \\
\hline $\mathrm{C} 10 \mathrm{~B}-\mathrm{C} 9 \mathrm{~B}-\mathrm{C} 8 \mathrm{~B}$ & $121.3(3)$ \\
\hline $\mathrm{C} 14 \mathrm{~B}-\mathrm{C} 9 \mathrm{~B}-\mathrm{C} 8 \mathrm{~B}$ & $120.0(3)$ \\
\hline $\mathrm{C} 14 \mathrm{~B}-\mathrm{C} 9 \mathrm{~B}-\mathrm{C} 10 \mathrm{~B}$ & $118.7(3)$ \\
\hline $\mathrm{C} 9 \mathrm{~B}-\mathrm{C} 10 \mathrm{~B}-\mathrm{H} 10 \mathrm{~B}$ & 120.1 \\
\hline $\mathrm{C} 11 \mathrm{~B}-\mathrm{C} 10 \mathrm{~B}-\mathrm{C} 9 \mathrm{~B}$ & $119.9(3)$ \\
\hline $\mathrm{C} 11 \mathrm{~B}-\mathrm{C} 10 \mathrm{~B}-\mathrm{H} 10 \mathrm{~B}$ & 120.1 \\
\hline $\mathrm{C} 10 \mathrm{~B}-\mathrm{C} 11 \mathrm{~B}-\mathrm{H} 11 \mathrm{~B}$ & 119.2 \\
\hline $\mathrm{C} 10 \mathrm{~B}-\mathrm{C} 11 \mathrm{~B}-\mathrm{C} 12 \mathrm{~B}$ & $121.5(3)$ \\
\hline $\mathrm{C} 12 \mathrm{~B}-\mathrm{C} 11 \mathrm{~B}-\mathrm{H} 11 \mathrm{~B}$ & 119.2 \\
\hline $\mathrm{C} 11 \mathrm{~B}-\mathrm{C} 12 \mathrm{~B}-\mathrm{C} 15 \mathrm{~B}$ & $120.0(3)$ \\
\hline $\mathrm{C} 13 \mathrm{~B}-\mathrm{C} 12 \mathrm{~B}-\mathrm{C} 11 \mathrm{~B}$ & $117.7(3)$ \\
\hline $\mathrm{C} 13 \mathrm{~B}-\mathrm{C} 12 \mathrm{~B}-\mathrm{C} 15 \mathrm{~B}$ & $122.3(3)$ \\
\hline $\mathrm{C} 12 \mathrm{~B}-\mathrm{C} 13 \mathrm{~B}-\mathrm{H} 13 \mathrm{~B}$ & 119.5 \\
\hline $\mathrm{C} 12 \mathrm{~B}-\mathrm{C} 13 \mathrm{~B}-\mathrm{C} 14 \mathrm{~B}$ & $121.1(3)$ \\
\hline $\mathrm{C} 14 \mathrm{~B}-\mathrm{C} 13 \mathrm{~B}-\mathrm{H} 13 \mathrm{~B}$ & 119.5 \\
\hline $\mathrm{C} 9 \mathrm{~B}-\mathrm{C} 14 \mathrm{~B}-\mathrm{C} 13 \mathrm{~B}$ & $121.1(3)$ \\
\hline $\mathrm{C} 9 \mathrm{~B}-\mathrm{C} 14 \mathrm{~B}-\mathrm{H} 14 \mathrm{~B}$ & 119.5 \\
\hline $\mathrm{C} 13 \mathrm{~B}-\mathrm{C} 14 \mathrm{~B}-\mathrm{H} 14 \mathrm{~B}$ & 119.5 \\
\hline $\mathrm{C} 12 \mathrm{~B}-\mathrm{C} 15 \mathrm{~B}-\mathrm{H} 15 \mathrm{D}$ & 109.5 \\
\hline $\mathrm{C} 12 \mathrm{~B}-\mathrm{C} 15 \mathrm{~B}-\mathrm{H} 15 \mathrm{E}$ & 109.5 \\
\hline $\mathrm{C} 12 \mathrm{~B}-\mathrm{C} 15 \mathrm{~B}-\mathrm{H} 15 \mathrm{~F}$ & 109.5 \\
\hline $\mathrm{H} 15 \mathrm{D}-\mathrm{C} 15 \mathrm{~B}-\mathrm{H} 15 \mathrm{E}$ & 109.5 \\
\hline $\mathrm{H} 15 \mathrm{D}-\mathrm{C} 15 \mathrm{~B}-\mathrm{H} 15 \mathrm{~F}$ & 109.5 \\
\hline $\mathrm{H} 15 \mathrm{E}-\mathrm{C} 15 \mathrm{~B}-\mathrm{H} 15 \mathrm{~F}$ & 109.5 \\
\hline $\mathrm{Na} 1 \mathrm{~A}-\mathrm{O} 1 \mathrm{~W}-\mathrm{Na} 1 \mathrm{~B}^{\mathrm{i}}$ & $81.89(7)$ \\
\hline $\mathrm{Na} 1 \mathrm{~A}-\mathrm{O} 1 \mathrm{~W}-\mathrm{H} 1 \mathrm{WA}$ & 120.2 \\
\hline $\mathrm{Na} 1 \mathrm{~A}-\mathrm{O} 1 \mathrm{~W}-\mathrm{H} 1 \mathrm{WB}$ & 105.3 \\
\hline Na1B $-\mathrm{O} 1 \mathrm{~W}-\mathrm{H} 1 \mathrm{WA}$ & 120.3 \\
\hline $\mathrm{Na}^{1} \mathrm{~B}^{\mathrm{i}}-\mathrm{O} 1 \mathrm{~W}-\mathrm{H} 1 \mathrm{WB}$ & 115.2 \\
\hline $\mathrm{H} 1 \mathrm{WA}-\mathrm{O} 1 \mathrm{~W}-\mathrm{H} 1 \mathrm{WB}$ & 110.6 \\
\hline $\mathrm{Na} 1 \mathrm{~A}-\mathrm{O} 2 \mathrm{~W}-\mathrm{Na} 1 \mathrm{~B}^{\mathrm{ii}}$ & $107.54(9)$ \\
\hline $\mathrm{Na} 1 \mathrm{~A}-\mathrm{O} 2 \mathrm{~W}-\mathrm{H} 2 \mathrm{WA}$ & 110.1 \\
\hline $\mathrm{Na} 1 \mathrm{~A}-\mathrm{O} 2 \mathrm{~W}-\mathrm{H} 2 \mathrm{WB}$ & 119.8 \\
\hline $\mathrm{Na}_{1} \mathrm{~B}^{\mathrm{ii}}-\mathrm{O} 2 \mathrm{~W}-\mathrm{H} 2 \mathrm{WA}$ & 97.1 \\
\hline $\mathrm{Na} 1 \mathrm{~B}^{\mathrm{ii}}-\mathrm{O} 2 \mathrm{~W}-\mathrm{H} 2 \mathrm{WB}$ & 115.5 \\
\hline
\end{tabular}




\begin{tabular}{|c|c|}
\hline $\mathrm{C} 12 \mathrm{~A}-\mathrm{C} 13 \mathrm{~A}-\mathrm{H} 13 \mathrm{~A}$ & 119.2 \\
\hline $\mathrm{C} 12 \mathrm{~A}-\mathrm{C} 13 \mathrm{~A}-\mathrm{C} 14 \mathrm{~A}$ & $121.5(3)$ \\
\hline $\mathrm{C} 14 \mathrm{~A}-\mathrm{C} 13 \mathrm{~A}-\mathrm{H} 13 \mathrm{~A}$ & 119.2 \\
\hline $\mathrm{C} 9 \mathrm{~A}-\mathrm{C} 14 \mathrm{~A}-\mathrm{H} 14 \mathrm{~A}$ & 119.7 \\
\hline $\mathrm{C} 13 \mathrm{~A}-\mathrm{C} 14 \mathrm{~A}-\mathrm{C} 9 \mathrm{~A}$ & $120.7(3)$ \\
\hline $\mathrm{C} 13 \mathrm{~A}-\mathrm{C} 14 \mathrm{~A}-\mathrm{H} 14 \mathrm{~A}$ & 119.7 \\
\hline $\mathrm{C} 12 \mathrm{~A}-\mathrm{C} 15 \mathrm{~A}-\mathrm{H} 15 \mathrm{~A}$ & 109.5 \\
\hline $\mathrm{C} 12 \mathrm{~A}-\mathrm{C} 15 \mathrm{~A}-\mathrm{H} 15 \mathrm{~B}$ & 109.5 \\
\hline $\mathrm{C} 12 \mathrm{~A}-\mathrm{C} 15 \mathrm{~A}-\mathrm{H} 15 \mathrm{C}$ & 109.5 \\
\hline $\mathrm{H} 15 \mathrm{~A}-\mathrm{C} 15 \mathrm{~A}-\mathrm{H} 15 \mathrm{~B}$ & 109.5 \\
\hline $\mathrm{Na} 1 \mathrm{~A}-\mathrm{O} 1 \mathrm{~A}-\mathrm{C} 1 \mathrm{~A}-\mathrm{O} 2 \mathrm{~A}$ & $21.2(3)$ \\
\hline $\mathrm{Na} 1 \mathrm{~A}-\mathrm{O} 1 \mathrm{~A}-\mathrm{C} 1 \mathrm{~A}-\mathrm{C} 2 \mathrm{~A}$ & $-155.9(2)$ \\
\hline $\mathrm{Na} 1 \mathrm{~A}-\mathrm{O} 2 \mathrm{~A}-\mathrm{C} 1 \mathrm{~A}-\mathrm{Na} 1 \mathrm{~A}^{\mathrm{i}}$ & $-53.7(6)$ \\
\hline $\mathrm{Na} 1 \mathrm{~A}-\mathrm{O} 2 \mathrm{~A}-\mathrm{C} 1 \mathrm{~A}-\mathrm{O} 1 \mathrm{~A}$ & $-19.5(3)$ \\
\hline $\mathrm{Na} 1 \mathrm{~A}-\mathrm{O} 2 \mathrm{~A}-\mathrm{C} 1 \mathrm{~A}-\mathrm{O} 1 \mathrm{~A}$ & $-73.2(8)$ \\
\hline $\mathrm{Na} 1 \mathrm{~A}-\mathrm{O} 2 \mathrm{~A}-\mathrm{C} 1 \mathrm{~A}-\mathrm{C} 2 \mathrm{~A}$ & $157.4(2)$ \\
\hline $\mathrm{Na} 1 \mathrm{~A}-\mathrm{O} 2 \mathrm{~A}-\mathrm{C} 1 \mathrm{~A}-\mathrm{C} 2 \mathrm{~A}$ & $103.7(7)$ \\
\hline $\mathrm{Na} 1 \mathrm{~A}-\mathrm{C} 1 \mathrm{~A}-\mathrm{C} 2 \mathrm{~A}-\mathrm{C} 3 \mathrm{~A}$ & $120.9(5)$ \\
\hline $\mathrm{O} 1 \mathrm{~A}-\mathrm{C} 1 \mathrm{~A}-\mathrm{C} 2 \mathrm{~A}-\mathrm{C} 3 \mathrm{~A}$ & $-167.4(3)$ \\
\hline $\mathrm{O} 2 \mathrm{~A}-\mathrm{C} 1 \mathrm{~A}-\mathrm{C} 2 \mathrm{~A}-\mathrm{C} 3 \mathrm{~A}$ & $15.5(4)$ \\
\hline $\mathrm{O} 3 \mathrm{~A}-\mathrm{C} 8 \mathrm{~A}-\mathrm{C} 9 \mathrm{~A}-\mathrm{C} 10 \mathrm{~A}$ & $-142.0(3)$ \\
\hline $\mathrm{O} 3 \mathrm{~A}-\mathrm{C} 8 \mathrm{~A}-\mathrm{C} 9 \mathrm{~A}-\mathrm{C} 14 \mathrm{~A}$ & $34.9(4)$ \\
\hline $\mathrm{N} 1 \mathrm{~A}-\mathrm{C} 3 \mathrm{~A}-\mathrm{C} 4 \mathrm{~A}-\mathrm{C} 5 \mathrm{~A}$ & $-0.8(3)$ \\
\hline $\mathrm{N} 1 \mathrm{~A}-\mathrm{C} 6 \mathrm{~A}-\mathrm{C} 8 \mathrm{~A}-\mathrm{O} 3 \mathrm{~A}$ & $15.4(5)$ \\
\hline $\mathrm{N} 1 \mathrm{~A}-\mathrm{C} 6 \mathrm{~A}-\mathrm{C} 8 \mathrm{~A}-\mathrm{C} 9 \mathrm{~A}$ & $-168.0(3)$ \\
\hline $\mathrm{C} 1 \mathrm{~A}-\mathrm{C} 2 \mathrm{~A}-\mathrm{C} 3 \mathrm{~A}-\mathrm{N} 1 \mathrm{~A}$ & $-70.1(4)$ \\
\hline $\mathrm{C} 1 \mathrm{~A}-\mathrm{C} 2 \mathrm{~A}-\mathrm{C} 3 \mathrm{~A}-\mathrm{C} 4 \mathrm{~A}$ & $110.7(3)$ \\
\hline $\mathrm{C} 2 \mathrm{~A}-\mathrm{C} 3 \mathrm{~A}-\mathrm{C} 4 \mathrm{~A}-\mathrm{C} 5 \mathrm{~A}$ & $178.5(3)$ \\
\hline $\mathrm{C} 3 \mathrm{~A}-\mathrm{N} 1 \mathrm{~A}-\mathrm{C} 6 \mathrm{~A}-\mathrm{C} 5 \mathrm{~A}$ & $-1.0(3)$ \\
\hline $\mathrm{C} 3 \mathrm{~A}-\mathrm{N} 1 \mathrm{~A}-\mathrm{C} 6 \mathrm{~A}-\mathrm{C} 8 \mathrm{~A}$ & $-168.7(3)$ \\
\hline $\mathrm{C} 3 \mathrm{~A}-\mathrm{C} 4 \mathrm{~A}-\mathrm{C} 5 \mathrm{~A}-\mathrm{C} 6 \mathrm{~A}$ & $0.2(3)$ \\
\hline $\mathrm{C} 4 \mathrm{~A}-\mathrm{C} 5 \mathrm{~A}-\mathrm{C} 6 \mathrm{~A}-\mathrm{N} 1 \mathrm{~A}$ & $0.5(3)$ \\
\hline $\mathrm{C} 4 \mathrm{~A}-\mathrm{C} 5 \mathrm{~A}-\mathrm{C} 6 \mathrm{~A}-\mathrm{C} 8 \mathrm{~A}$ & $167.7(3)$ \\
\hline $\mathrm{C} 5 \mathrm{~A}-\mathrm{C} 6 \mathrm{~A}-\mathrm{C} 8 \mathrm{~A}-\mathrm{O} 3 \mathrm{~A}$ & $-149.7(3)$ \\
\hline $\mathrm{C} 5 \mathrm{~A}-\mathrm{C} 6 \mathrm{~A}-\mathrm{C} 8 \mathrm{~A}-\mathrm{C} 9 \mathrm{~A}$ & $26.9(4)$ \\
\hline $\mathrm{C} 6 \mathrm{~A}-\mathrm{N} 1 \mathrm{~A}-\mathrm{C} 3 \mathrm{~A}-\mathrm{C} 2 \mathrm{~A}$ & $-178.3(2)$ \\
\hline $\mathrm{C} 6 \mathrm{~A}-\mathrm{N} 1 \mathrm{~A}-\mathrm{C} 3 \mathrm{~A}-\mathrm{C} 4 \mathrm{~A}$ & $1.1(3)$ \\
\hline $\mathrm{C} 6 \mathrm{~A}-\mathrm{C} 8 \mathrm{~A}-\mathrm{C} 9 \mathrm{~A}-\mathrm{C} 10 \mathrm{~A}$ & $41.3(4)$ \\
\hline $\mathrm{C} 6 \mathrm{~A}-\mathrm{C} 8 \mathrm{~A}-\mathrm{C} 9 \mathrm{~A}-\mathrm{C} 14 \mathrm{~A}$ & $-141.8(3)$ \\
\hline $\mathrm{C} 7 \mathrm{~A}-\mathrm{N} 1 \mathrm{~A}-\mathrm{C} 3 \mathrm{~A}-\mathrm{C} 2 \mathrm{~A}$ & $-6.0(4)$ \\
\hline $\mathrm{C} 7 \mathrm{~A}-\mathrm{N} 1 \mathrm{~A}-\mathrm{C} 3 \mathrm{~A}-\mathrm{C} 4 \mathrm{~A}$ & $173.4(2)$ \\
\hline $\mathrm{C} 7 \mathrm{~A}-\mathrm{N} 1 \mathrm{~A}-\mathrm{C} 6 \mathrm{~A}-\mathrm{C} 5 \mathrm{~A}$ & $-173.2(2)$ \\
\hline $\mathrm{C} 7 \mathrm{~A}-\mathrm{N} 1 \mathrm{~A}-\mathrm{C} 6 \mathrm{~A}-\mathrm{C} 8 \mathrm{~A}$ & $19.0(4)$ \\
\hline $\mathrm{C} 8 \mathrm{~A}-\mathrm{C} 9 \mathrm{~A}-\mathrm{C} 10 \mathrm{~A}-\mathrm{C} 11 \mathrm{~A}$ & $178.7(3)$ \\
\hline $\mathrm{C} 8 \mathrm{~A}-\mathrm{C} 9 \mathrm{~A}-\mathrm{C} 14 \mathrm{~A}-\mathrm{C} 13 \mathrm{~A}$ & $-179.9(3)$ \\
\hline $\mathrm{C} 9 \mathrm{~A}-\mathrm{C} 10 \mathrm{~A}-\mathrm{C} 11 \mathrm{~A}-\mathrm{C} 12 \mathrm{~A}$ & $-0.5(5)$ \\
\hline $\mathrm{C} 10 \mathrm{~A}-\mathrm{C} 9 \mathrm{~A}-\mathrm{C} 14 \mathrm{~A}-\mathrm{C} 13 \mathrm{~A}$ & $-2.8(5)$ \\
\hline
\end{tabular}

H2WA-O2W-H2WB $\quad 104.2$

Na1B-O3W-H3WA

$\mathrm{Na} 1 \mathrm{~B}-\mathrm{O} 3 \mathrm{~W}-\mathrm{H} 3 \mathrm{WB}$

H3WA-O3W-H3WB

Na1A $-\mathrm{O} 4 \mathrm{~W}-\mathrm{H} 4 \mathrm{WA}$

Na1A - O4W-H4WB

$\mathrm{Na} 1 \mathrm{~B}-\mathrm{O} 4 \mathrm{~W}-\mathrm{Na} 1 \mathrm{~A}^{\mathrm{i}}$

Na1B-O 4 W-H4WA

Na1B-O 4 W-H4WB

$\mathrm{H} 4 \mathrm{WA}-\mathrm{O} 4 \mathrm{~W}-\mathrm{H} 4 \mathrm{WB}$

$\mathrm{C} 15 \mathrm{~A}-\mathrm{C} 12 \mathrm{~A}-\mathrm{C} 13 \mathrm{~A}-\mathrm{C} 14 \mathrm{~A}$

$\mathrm{Na} 1 \mathrm{~B}-\mathrm{O} 1 \mathrm{~A}-\mathrm{C} 1 \mathrm{~A}-\mathrm{Na} 1 \mathrm{~A}^{\mathrm{i}}$

$\mathrm{Na} 1 \mathrm{~B}-\mathrm{O} 1 \mathrm{~A}-\mathrm{C} 1 \mathrm{~A}-\mathrm{O} 2 \mathrm{~A}$

$\mathrm{Na} 1 \mathrm{~B}-\mathrm{O} 1 \mathrm{~A}-\mathrm{C} 1 \mathrm{~A}-\mathrm{C} 2 \mathrm{~A}$

$\mathrm{Na} 1 \mathrm{~B}-\mathrm{O} 1 \mathrm{~B}-\mathrm{C} 1 \mathrm{~B}-\mathrm{O} 2 \mathrm{~B}$

$\mathrm{Na} 1 \mathrm{~B}-\mathrm{O} 1 \mathrm{~B}-\mathrm{C} 1 \mathrm{~B}-\mathrm{C} 2 \mathrm{~B}$

$\mathrm{O} 1 \mathrm{~B}-\mathrm{C} 1 \mathrm{~B}-\mathrm{C} 2 \mathrm{~B}-\mathrm{C} 3 \mathrm{~B}$

$\mathrm{O} 2 \mathrm{~B}-\mathrm{C} 1 \mathrm{~B}-\mathrm{C} 2 \mathrm{~B}-\mathrm{C} 3 \mathrm{~B}$

$\mathrm{O} 3 \mathrm{~B}-\mathrm{C} 8 \mathrm{~B}-\mathrm{C} 9 \mathrm{~B}-\mathrm{C} 10 \mathrm{~B}$

$\mathrm{O} 3 \mathrm{~B}-\mathrm{C} 8 \mathrm{~B}-\mathrm{C} 9 \mathrm{~B}-\mathrm{C} 14 \mathrm{~B}$

$\mathrm{N} 1 \mathrm{~B}-\mathrm{C} 3 \mathrm{~B}-\mathrm{C} 4 \mathrm{~B}-\mathrm{C} 5 \mathrm{~B}$

$\mathrm{N} 1 \mathrm{~B}-\mathrm{C} 6 \mathrm{~B}-\mathrm{C} 8 \mathrm{~B}-\mathrm{O} 3 \mathrm{~B}$

$\mathrm{N} 1 \mathrm{~B}-\mathrm{C} 6 \mathrm{~B}-\mathrm{C} 8 \mathrm{~B}-\mathrm{C} 9 \mathrm{~B}$

$\mathrm{C} 1 \mathrm{~B}-\mathrm{C} 2 \mathrm{~B}-\mathrm{C} 3 \mathrm{~B}-\mathrm{N} 1 \mathrm{~B}$

$\mathrm{C} 1 \mathrm{~B}-\mathrm{C} 2 \mathrm{~B}-\mathrm{C} 3 \mathrm{~B}-\mathrm{C} 4 \mathrm{~B}$

$\mathrm{C} 2 \mathrm{~B}-\mathrm{C} 3 \mathrm{~B}-\mathrm{C} 4 \mathrm{~B}-\mathrm{C} 5 \mathrm{~B}$

$\mathrm{C} 3 \mathrm{~B}-\mathrm{N} 1 \mathrm{~B}-\mathrm{C} 6 \mathrm{~B}-\mathrm{C} 5 \mathrm{~B}$

$\mathrm{C} 3 \mathrm{~B}-\mathrm{N} 1 \mathrm{~B}-\mathrm{C} 6 \mathrm{~B}-\mathrm{C} 8 \mathrm{~B}$

$\mathrm{C} 3 \mathrm{~B}-\mathrm{C} 4 \mathrm{~B}-\mathrm{C} 5 \mathrm{~B}-\mathrm{C} 6 \mathrm{~B}$

$\mathrm{C} 4 \mathrm{~B}-\mathrm{C} 5 \mathrm{~B}-\mathrm{C} 6 \mathrm{~B}-\mathrm{N} 1 \mathrm{~B}$

$\mathrm{C} 4 \mathrm{~B}-\mathrm{C} 5 \mathrm{~B}-\mathrm{C} 6 \mathrm{~B}-\mathrm{C} 8 \mathrm{~B}$

$\mathrm{C} 5 \mathrm{~B}-\mathrm{C} 6 \mathrm{~B}-\mathrm{C} 8 \mathrm{~B}-\mathrm{O} 3 \mathrm{~B}$

$\mathrm{C} 5 \mathrm{~B}-\mathrm{C} 6 \mathrm{~B}-\mathrm{C} 8 \mathrm{~B}-\mathrm{C} 9 \mathrm{~B}$

$\mathrm{C} 6 \mathrm{~B}-\mathrm{N} 1 \mathrm{~B}-\mathrm{C} 3 \mathrm{~B}-\mathrm{C} 2 \mathrm{~B}$

$\mathrm{C} 6 \mathrm{~B}-\mathrm{N} 1 \mathrm{~B}-\mathrm{C} 3 \mathrm{~B}-\mathrm{C} 4 \mathrm{~B}$

$\mathrm{C} 6 \mathrm{~B}-\mathrm{C} 8 \mathrm{~B}-\mathrm{C} 9 \mathrm{~B}-\mathrm{C} 10 \mathrm{~B}$

$\mathrm{C} 6 \mathrm{~B}-\mathrm{C} 8 \mathrm{~B}-\mathrm{C} 9 \mathrm{~B}-\mathrm{C} 14 \mathrm{~B}$

$\mathrm{C} 7 \mathrm{~B}-\mathrm{N} 1 \mathrm{~B}-\mathrm{C} 3 \mathrm{~B}-\mathrm{C} 2 \mathrm{~B}$

$\mathrm{C} 7 \mathrm{~B}-\mathrm{N} 1 \mathrm{~B}-\mathrm{C} 3 \mathrm{~B}-\mathrm{C} 4 \mathrm{~B}$

$\mathrm{C} 7 \mathrm{~B}-\mathrm{N} 1 \mathrm{~B}-\mathrm{C} 6 \mathrm{~B}-\mathrm{C} 5 \mathrm{~B}$

$\mathrm{C} 7 \mathrm{~B}-\mathrm{N} 1 \mathrm{~B}-\mathrm{C} 6 \mathrm{~B}-\mathrm{C} 8 \mathrm{~B}$

$\mathrm{C} 8 \mathrm{~B}-\mathrm{C} 9 \mathrm{~B}-\mathrm{C} 10 \mathrm{~B}-\mathrm{C} 11 \mathrm{~B}$

$\mathrm{C} 8 \mathrm{~B}-\mathrm{C} 9 \mathrm{~B}-\mathrm{C} 14 \mathrm{~B}-\mathrm{C} 13 \mathrm{~B}$

$\mathrm{C} 9 \mathrm{~B}-\mathrm{C} 10 \mathrm{~B}-\mathrm{C} 11 \mathrm{~B}-\mathrm{C} 12 \mathrm{~B}$

$\mathrm{C} 10 \mathrm{~B}-\mathrm{C} 9 \mathrm{~B}-\mathrm{C} 14 \mathrm{~B}-\mathrm{C} 13 \mathrm{~B}$

$\mathrm{C} 10 \mathrm{~B}-\mathrm{C} 11 \mathrm{~B}-\mathrm{C} 12 \mathrm{~B}-\mathrm{C} 13 \mathrm{~B}$

$\mathrm{C} 10 \mathrm{~B}-\mathrm{C} 11 \mathrm{~B}-\mathrm{C} 12 \mathrm{~B}-\mathrm{C} 15 \mathrm{~B}$
110.1

111.9

106.7

118.6

139.0

$82.42(7)$

117.6

105.9

93.5

$-178.7(3)$

$-85.19(19)$

$-64.0(4)$

$119.0(2)$

40.8 (4)

$-141.7(2)$

$170.7(3)$

$-11.7(4)$

$144.1(3)$

$-34.1(4)$

$2.0(3)$

$-16.1(5)$

$166.3(3)$

$71.0(4)$

$-105.8(3)$

$179.2(3)$

$1.6(3)$

$169.0(3)$

$-1.1(4)$

-0.3 (3)

-167.0 (3)

$148.6(3)$

-29.0 (4)

-179.5 (3)

-2.2 (3)

-38.2 (4)

$143.6(3)$

6.3 (4)

-176.4 (3)

175.7 (3)

-16.9 (4)

-180.0 (3)

-179.1 (3)

0.3 (5)

2.7 (5)

0.3 (5)

-178.3 (3) 


$\begin{array}{llll}\mathrm{C} 10 \mathrm{~A}-\mathrm{C} 11 \mathrm{~A}-\mathrm{C} 12 \mathrm{~A}-\mathrm{C} 13 \mathrm{~A} & 0.3(5) & \mathrm{C} 11 \mathrm{~B}-\mathrm{C} 12 \mathrm{~B}-\mathrm{C} 13 \mathrm{~B}-\mathrm{C} 14 \mathrm{~B} & 0.6(5) \\ \mathrm{C} 10 \mathrm{~A}-\mathrm{C} 11 \mathrm{~A}-\mathrm{C} 12 \mathrm{~A}-\mathrm{C} 15 \mathrm{~A} & 177.7(3) & \mathrm{C} 12 \mathrm{~B}-\mathrm{C} 13 \mathrm{~B}-\mathrm{C} 14 \mathrm{~B}-\mathrm{C} 9 \mathrm{~B} & -2.1(5) \\ \mathrm{C} 11 \mathrm{~A}-\mathrm{C} 12 \mathrm{~A}-\mathrm{C} 13 \mathrm{~A}-\mathrm{C} 14 \mathrm{~A} & -1.3(5) & \mathrm{C} 14 \mathrm{~B}-\mathrm{C} 9 \mathrm{~B}-\mathrm{C} 10 \mathrm{~B}-\mathrm{C} 11 \mathrm{~B} & -1.8(5) \\ \mathrm{C} 12 \mathrm{~A}-\mathrm{C} 13 \mathrm{~A}-\mathrm{C} 14 \mathrm{~A}-\mathrm{C} 9 \mathrm{~A} & 2.7(5) & \mathrm{C} 15 \mathrm{~B}-\mathrm{C} 12 \mathrm{~B}-\mathrm{C} 13 \mathrm{~B}-\mathrm{C} 14 \mathrm{~B} & 179.1(3) \\ \mathrm{C} 14 \mathrm{~A}-\mathrm{C} 9 \mathrm{~A}-\mathrm{C} 10 \mathrm{~A}-\mathrm{C} 11 \mathrm{~A} & 1.8(4) & & \end{array}$

Symmetry codes: (i) $-x+2,-y+1,-z+1$; (ii) $x+1, y, z$; (iii) $x-1, y, z$.

Hydrogen-bond geometry $\left(\AA,{ }^{\circ}\right)$

\begin{tabular}{lllll}
\hline$D-\mathrm{H} \cdots A$ & $D-\mathrm{H}$ & $\mathrm{H} \cdots A$ & $D \cdots A$ & $D-\mathrm{H} \cdots A$ \\
\hline $\mathrm{O} 1 W-\mathrm{H} 1 W A \cdots \mathrm{O} 3 A$ & 0.89 & 2.12 & $3.002(3)$ & 169 \\
$\mathrm{O} 1 W-\mathrm{H} 1 W B \cdots \mathrm{O} 1 A^{\mathrm{ii}}$ & 0.89 & 2.00 & $2.665(4)$ & 130 \\
$\mathrm{O} 2 W-\mathrm{H} 2 W A \cdots \mathrm{O} 2 B^{\mathrm{ii}}$ & 0.89 & 1.88 & $2.741(3)$ & 161 \\
$\mathrm{O} 2 W-\mathrm{H} 2 W B \cdots \mathrm{O} 2 B^{\mathrm{iv}}$ & 0.89 & 2.13 & $3.019(3)$ & 172 \\
$\mathrm{O} 3 W-\mathrm{H} 3 W A \cdots \mathrm{O} 3 B^{\mathrm{v}}$ & 0.89 & 2.16 & $2.961(3)$ & 150 \\
$\mathrm{O} 3 W-\mathrm{H} 3 W B \cdots \mathrm{O} 1 B^{\mathrm{iv}}$ & 0.89 & 1.84 & $2.699(4)$ & 161 \\
$\mathrm{O} 4 W-\mathrm{H} 4 W A \cdots \mathrm{O} 1 B^{\text {iv }}$ & 0.89 & 2.19 & $2.894(3)$ & 136 \\
$\mathrm{O} 4 W-\mathrm{H} 4 W B \cdots \mathrm{O} 3 W^{\text {iv }}$ & 0.89 & 2.02 & $2.889(3)$ & 163 \\
\hline
\end{tabular}

Symmetry codes: (ii) $x+1, y, z$; (iv) $-x+1,-y,-z+1$; (v) $-x,-y,-z+1$. 